ALEA, Lat. Am. J. Probab. Math. Stat. 18, 761-792 (2021)

DOI: 10.30757/ALEA.v18-28

\title{
BSDEs with jumps and two completely separated irregular barriers in a general filtration
}

\author{
Mohamed Marzougue and Mohamed El Otmani \\ Ibn Zohr University \\ Laboratory of Analysis and Applied Mathematics (LAMA), \\ Faculty of sciences Agadir \\ Agadir, Morocco. \\ E-mail address: mohamed.marzougue@edu.uiz.ac.ma, m.elotmani@uiz.ac.ma
}

\begin{abstract}
We consider a doubly reflected backward stochastic differential equations with jumps and two completely separated optional barriers in a general filtration that supports a one-dimensional Brownian motion and an independent Poisson random measure. We suppose that the barriers have trajectories with left and right finite limits. We provide the existence and uniqueness result when the coefficient is stochastic Lipschitz by using a penalization method.
\end{abstract}

\section{Introduction}

Backward Stochastic Differential Equations (BSDEs in short) were introduced (in the linear case) by Bismut (1973). The non-linear case was developed by Pardoux and Peng (1990). These equations have attracted great interest due to their connections with mathematical finance (e.g., El Karoui et al., 1997c; El Karoui and Quenez, 1997), stochastic control and stochastic games (e.g., Bismut, 1973) and partial differential equations (e.g., Pardoux and Peng, 1992). Further, El Karoui et al. (1997a) have introduced the notion of reflected BSDEs (RBSDEs in short), which is a BSDE but the solution is forced to stay above a given process called barrier (or obstacle). Once more under square integrability of the terminal condition and the barrier and Lipschitz property on the coefficient, the authors have proved the existence and uniqueness results in the case of a Brownian filtration and a continuous barrier. Later, there have been several extensions of this work to the case of a discontinuous barrier (see for example Hamadène, 2002; Hamadène

Received by the editors September 12th, 2019; accepted January 26th, 2021.

2010 Mathematics Subject Classification. 60H05, 60H20.

Key words and phrases. Doubly reflected backward stochastic differential equations, optional barriers, general filtration, stochastic Lipschitz coefficient, penalization method.

This research was supported by the National Center for Scientific and Technical Research (CNRST), Morocco.

Corresponding author: Mohamed Marzougue. 
and Ouknine, 2003, 2016; Essaky, 2008). In all the above-mentioned works on RBSDEs, an assumption of right continuity of the barrier is made. Furthermore, Grigorova et al. (2017) have presented a new extension of the theory of RBSDEs to the case where the barrier is not necessarily right-continuous "just right uppersemicontinuous (r.u.s.c in short)". In this work, the authors have studied the existence and uniqueness result under the Lipschitz assumption on the coefficient. On the other hand, Baadi and Ouknine $(2017,2018)$ have considered the case of RBSDEs with r.u.s.c barrier in a general filtration. The more general case, when we do not make any regularity assumptions on the barrier, has been studied by Grigorova et al. (2020). Recently, Marzougue and El Otmani (2019) have discussed RBSDEs with r.u.s.c barrier under the so-called stochastic Lipschitz coefficient introduced by El Karoui and Huang (1997). On the other hand, Klimsiak et al. (2019) have proved that the solution of RBSDEs with optional barrier may be approximate by a modified penalization method. Very recently, Marzougue and El Otmani (2020a) have discussed the case of RBSDEs with general filtration where the barrier is assumed to be predictable and not necessarily right-continuous. Another interested work of RBSDEs with optional semimartingale barrier has been studied by Akdim et al. (2020). It is well known that RBSDEs have been proven to be powerful tools in mathematical finance (e.g., El Karoui et al., 1997b), mixed game problems (e.g., Hamadène and Lepeltier, 2000), providing a probabilistic formula for the viscosity solution of an obstacle problem for a class of parabolic partial differential equations (e.g., El Karoui et al., 1997a) and dynamic risk measures (e.g., Quenez and Sulem, 2014).

Doubly reflected BSDEs (DRBSDEs in short) have been introduced by Cvitanić and Karatzas (1996) in the case of continuous barriers, a Brownian filtration and a Lipschitz coefficient. The solutions of such equations are constrained to stay the first component of solutions between the lower barrier $\xi$ and the upper barrier $\zeta$. Many efforts have been made to relax the assumptions on the coefficient and barriers, namely Bahlali et al. (2005); Essaky et al. (2005); Hamadène and Hassani (2005, 2006); Li and Shi (2016); Marzougue and El Otmani (2017) and so on and so forth. In the case of discontinuous barriers, Hamadène and Wang (2009) have showed the existence and the uniqueness of a solution when the barriers and their left limits are completely separated. Recently, Marzougue and El Otmani (2020b) have established the existence and uniqueness of the solution to DRBSDEs with jumps and right-continuous completely separated barriers under stochastic Lipschitz coefficient. Later, Grigorova et al. (2018) have formulated a notion of DRBSDEs where the barriers are not necessarily right-continuous. The authors have showed the existence and uniqueness of the solution of these equations under the so-called Mokobodski's condition (assuming the existence of two strong supermartingales whose difference is between $\xi$ and $\zeta$ ) and a Lipschitz driver in a general filtration. Recently, Marzougue and El Otmani (2018) have obtained the corresponding existence and uniqueness results of DRBSDEs studied by Grigorova et al. (2018) but under stochastic Lipschitz coefficient. Very recently, Arharas et al. (2021) have discussed the case of DRBSDEs with general filtration where the barriers are assumed to be predictable and not necessarily right-continuous. On the other hand, Klimsiak et al. (2020) have proved that the solution of DRBSDEs with optional barriers may be approximate by a modified penalization method. 
As application, Grigorova et al. (2018) have proved that if $\xi$ and $-\zeta$ are r.u.s.c then there exists a (common) value function for the corresponding $\mathcal{E}^{f}$-Dynkin game problem, that is

$$
\mathbf{V}(\theta)=\underset{\nu \in \mathcal{T}_{[\theta, T]}}{\operatorname{essinf}} \operatorname{ess} \sup \mathcal{T}_{[\theta, T]}^{f} \mathcal{E}_{\theta, \tau \wedge \nu}[J(\tau, \nu)]=\underset{\tau \in \mathcal{T}_{[\theta, T]}}{\operatorname{ess} \sup } \underset{\nu \in \mathcal{T}_{[\theta, T]}}{\operatorname{essinf}} \mathcal{E}_{\theta, \tau \wedge \nu}^{f}[J(\tau, \nu)]
$$

where $\mathcal{E}_{t, \tau \wedge \nu}^{f}[$.$] denotes the non-linear f$-expectation at time $t$ where the terminal time is $\tau \wedge \nu, J(\tau, \nu)$ is the terminal payoff of the game (at stopping time $\tau \wedge \nu$ ) and $\mathcal{T}_{[\theta, T]}$ is the collection of all stopping times $\tau$ with values between $\theta$ and $T(T>0$ is a fixed horizon).

In the general case where the barriers do not satisfy any regularity assumptions, the solution of the DRBSDE is related to the value of "an extension" of the previous non-linear game problem over a larger set of "stopping strategies" than the set of stopping times. In this context, Grigorova et al. (2018) introduced the notion of stopping system which is an example of divided stopping time in the sense of El Karoui (1981) and the recent work of Marzougue (2020). Briefly, we recall the definition of stopping system: given $\tau \in \mathcal{T}_{[0, T]}$ be a stopping time and $H$ be a set of $\mathcal{F}_{\tau}$, we say that $\rho:=(\tau, H)$ be a stopping system if $H^{c} \cap\{\tau=T\}=\emptyset$ where $H^{c}$ denote the complement of $H$ in $\Omega$. Now, let us have a look at the zero-sum game problem where the set of "stopping strategies" of the agents is the set of stopping systems. More precisely, for two stopping systems $\rho:=(\tau, H)$ and $\rho^{\prime}:=\left(\nu, H^{\prime}\right)$, we define the payoff $J\left(\rho, \rho^{\prime}\right)$ by

$$
J\left(\rho, \rho^{\prime}\right)=\xi_{\rho}^{u} \mathbb{1}_{\{\tau \leq \nu\}}+\zeta_{\rho^{\prime}}^{l} \mathbb{1}_{\{\nu<\tau\}}
$$

with $\xi_{\rho}^{u}:=\xi_{\tau} \mathbb{1}_{H}+\hat{\xi}_{\tau} \mathbb{1}_{H^{c}}$ and $\zeta_{\rho^{\prime}}^{l}:=\zeta_{\nu} \mathbb{1}_{H^{\prime}}+\breve{\zeta}_{\nu} \mathbb{1}_{H^{\prime c}}$ where $\hat{\xi}$ denote the right uppersemicontinuous envelope of $\xi$ (i.e. $\hat{\xi}_{t}:=\limsup _{s \downarrow t, s>t} \xi_{s}$ ) and $\breve{\zeta}$ denote the right lower-semicontinuous envelope of $\zeta$ (i.e. $\breve{\zeta}_{t}:=\liminf _{s \downarrow t, s>t} \zeta_{s}$ ). From Grigorova et al. (2018), the payoff is assessed by an $f$-expectation, where $f$ is a Lipschitz driver, and the $\mathcal{E}^{f}$-Dynkin game (over the set of stopping systems) has a unique (common) value function, that is

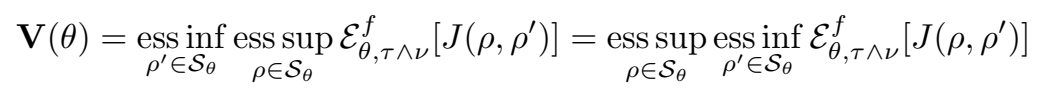

where $\mathcal{S}_{\theta}$ is the set of all stopping systems $\rho:=(\tau, H)$ such that $\theta \leq \tau$. An interesting question is how to be the value function of the $\mathcal{E}^{f}$-Dynkin game when $f$ is given stochastic Lipschitz. To this aim, since the value of $\mathcal{E}^{f}$-Dynkin game is interpreting by the solution of the DRBSDEs, we purpose to studying DRBSDEs with jumps and two completely separated irregular barriers under a stochastic Lipschitz coefficient in a general filtration (not necessarily quasi left-continuous). We show the existence and uniqueness of the solution by using a penalization method. Moreover, we investigate a comparison theorem for the solutions.

The paper is organized as follows: In Section 2, we give some notations and assumptions needed in this paper, and we define our DRBSDE. Section 3 is devoted to solve our DRBSDEs under our assumptions supposed for the data. In Section 4, we give the comparison theorem for the solutions of DRBSDEs. 


\section{Preliminaries}

Let $T$ strictly positive real number. Let $\left(\Omega, \mathcal{F},\left(\mathcal{F}_{t}\right)_{t \leq T}, \mathbb{P}\right)$ be a filtered probability space. The filtration $\left(\mathcal{F}_{t}\right)_{t \leq T}$ is assumed to be complete, right continuous and generated by one-dimensional Brownian motion $\left(B_{t}\right)_{t \leq T}$ and an independent Poisson random measure $\mu(d t, d e)$ with compensator $\lambda(d e) d t$. We denote by $\tilde{\mu}(d t, d e)$ the compensated process, i.e. $\tilde{\mu}(d t, d e):=\mu(d t, d e)-\lambda(d e) d t$. Let $(\mathcal{U}, \mathrm{U})$ be a measurable space equipped with a $\sigma$-finite positive measure $\lambda$. We will denote by

- |.| the Euclidian norm on $\mathbb{R}^{d}$.

- $\mathcal{T}_{[t, T]}$ the set of stopping times $\tau$ such that $\tau \in[t, T]$.

- $\mathcal{P}$ (resp. Prog) the predictable (resp. progressive) $\sigma$-algebra on $\Omega \times[0, T]$.

- $\mathcal{B}\left(\mathbb{R}^{d}\right)$ the Borelian $\sigma$-algebra on $\mathbb{R}^{d}$.

- $\mathcal{L}_{\lambda}$ the set of $\mathrm{U} \otimes \mathcal{B}(\mathbb{R})$-measurable mapping $V: \mathcal{U} \rightarrow \mathbb{R}$ such that $\|V\|_{\lambda}^{2}=$ $\int_{\mathcal{U}}|V(e)|^{2} \lambda(d e)<+\infty$.

- $\mathcal{B}\left(\mathcal{L}_{\lambda}\right)$ the Borelian $\sigma$-algebra on $\mathcal{L}_{\lambda}$.

- $\mathcal{M}$ the set of càdlàg local martingales orthogonal with respect to $B$ and $\tilde{\mu}$, i.e. if $M \in \mathcal{M}$ then $\langle M, B\rangle$. $=0$ and $\left\langle M, \int_{0}^{\cdot} \int_{\mathcal{U}} V_{s}(e) \tilde{\mu}(d s, d e)\right\rangle$. $=0$ for all $V \in \mathcal{L}_{\lambda}$.

Let's introduce some spaces:

- $\mathcal{M}^{2}$ is the subspace of $\mathcal{M}$ of all martingales $\left(M_{t}\right)_{t \leq T}$ such that

$$
\|M\|_{\mathcal{M}^{2}}^{2}=\mathbb{E}\langle M\rangle_{T}=\mathbb{E}[M]_{T}<+\infty .
$$

- $\mathcal{H}^{2}$ is the space of $\mathbb{R}$-valued and predictable processes $\left(Z_{t}\right)_{t \leq T}$ such that

$$
\|Z\|_{\mathcal{H}^{2}}^{2}=\mathbb{E}\left[\int_{0}^{T}\left|Z_{t}\right|^{2} d t\right]<+\infty .
$$

- $\mathcal{S}^{2}$ is the space of $\mathbb{R}$-valued and optional processes $\left(K_{t}\right)_{t \leq T}$ such that

$$
\|K\|_{\mathcal{S}^{2}}^{2}=\mathbb{E}\left[\operatorname{ess}_{\tau \in \mathcal{T}_{[0, T]}}^{\operatorname{esup}}\left|K_{\tau}\right|^{2}\right]<+\infty .
$$

- $\mathcal{L}^{2}$ is the space of $\mathbb{R}$-valued and $(\mathcal{P} \otimes \mathrm{U}, \mathcal{B}(\mathbb{R}))$-measurable processes $V$ : $\Omega \times[0, T] \times \mathcal{U} \rightarrow \mathbb{R}$ such that

$$
\|V\|_{\mathcal{L}^{2}}^{2}=\mathbb{E}\left[\int_{0}^{T}\left\|V_{t}\right\|_{\lambda}^{2} d t\right]<+\infty .
$$

Let $\beta>0$ and $\left(a_{t}\right)_{t \leq T}$ be a nonnegative $\mathcal{F}_{t}$-adapted process. We define the increasing continuous process $A_{t}:=\int_{0}^{t} a_{s}^{2} d s$, for all $t \leq T$ and we introduce the following spaces :

- $\mathcal{S}_{\beta}^{2}$ is the space of $\mathbb{R}$-valued and optional processes $\left(Y_{t}\right)_{t \leq T}$ such that

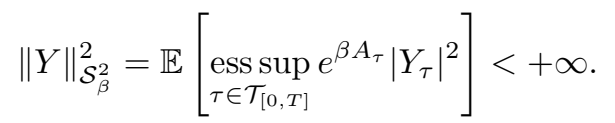

- $\mathcal{S}_{\beta}^{2, A}$ is the space of $\mathbb{R}$-valued and optional processes $\left(Y_{t}\right)_{t \leq T}$ such that

$$
\|Y\|_{\mathcal{S}_{\beta}^{2, A}}^{2}=\mathbb{E}\left[\int_{0}^{T} e^{\beta A_{t}}\left|Y_{t}\right|^{2} d A_{t}\right]<+\infty .
$$


- $\mathcal{H}_{\beta}^{2}$ is the space of $\mathbb{R}$-valued and predictable processes $\left(Z_{t}\right)_{t \leq T}$ such that

$$
\|Z\|_{\mathcal{H}_{\beta}^{2}}^{2}=\mathbb{E}\left[\int_{0}^{T} e^{\beta A_{t}}\left|Z_{t}\right|^{2} d t\right]<+\infty .
$$

- $\mathcal{L}_{\beta}^{2}$ is the space of $\mathbb{R}$-valued and $(\mathcal{P} \otimes U, \mathcal{B}(\mathbb{R}))$-measurable processes $V$ : $\Omega \times[0, T] \times \mathcal{U} \rightarrow \mathbb{R}$ such that

$$
\|V\|_{\mathcal{L}_{\beta}^{2}}^{2}=\mathbb{E}\left[\int_{0}^{T} e^{\beta A_{t}}\left\|V_{t}\right\|_{\lambda}^{2} d t\right]<+\infty .
$$

- $\mathcal{M}_{\beta}^{2}$ is the subspace of $\mathcal{M}^{2}$ of all càdlàg martingales $\left(M_{t}\right)_{t \leq T}$ such that

$$
\|M\|_{\mathcal{M}_{\beta}^{2}}^{2}=\mathbb{E} \int_{0}^{T} e^{\beta A_{t}} d[M]_{t}<+\infty .
$$

- $\mathfrak{B}_{\beta}^{2}=\mathcal{S}_{\beta}^{2} \cap \mathcal{S}_{\beta}^{2, A}$.

A function $f$ is said to be a stochastic Lipschitz driver if

(i) $f: \Omega \times[0, T] \times \mathbb{R} \times \mathbb{R} \times \mathcal{L}_{\lambda} \longrightarrow \mathbb{R},(\omega, t, y, z, v) \mapsto f(\omega, t, y, z, v)$ is Prog $\otimes$ $\mathcal{B}(\mathbb{R}) \otimes \mathcal{B}\left(\mathcal{L}_{\lambda}\right)$-measurable.

(ii) There exist three nonnegative $\mathcal{F}_{t}$-adapted processes $\left(\theta_{t}\right)_{t \leq T},\left(\gamma_{t}\right)_{t \leq T}$ and $\left(\eta_{t}\right)_{t \leq T}$ such that for all $\left(t, y, y^{\prime}, z, z^{\prime}, v, v^{\prime}\right) \in[0, T] \times \mathbb{R}^{4} \times \mathcal{L}_{\lambda} \times \mathcal{L}_{\lambda}$

$$
\left|f(t, y, z, v)-f\left(t, y^{\prime}, z^{\prime}, v^{\prime}\right)\right| \leq \theta_{t}\left|y-y^{\prime}\right|+\gamma_{t}\left|z-z^{\prime}\right|+\eta_{t}\left\|v-v^{\prime}\right\|_{\lambda} .
$$

(iii) For all $t \in[0, T]$ there exists $\epsilon>0$ such that $a_{t}^{2}:=\theta_{t}+\gamma_{t}^{2}+\eta_{t}^{2} \geq \epsilon$.

(iv) One has

$$
\forall t \in[0, T], \quad \frac{f(t, 0,0,0)}{a_{t}} \in \mathcal{H}_{\beta}^{2} .
$$

A process $\mathcal{Y}: \Omega \times[0, T] \rightarrow \mathbb{R}$ is said to be regulated process (or we say: $\mathcal{Y}$ has regulated trajectories) if $\mathcal{Y}$ has a left limit in each point of $] 0, T]$, and a right limit in each point of $[0, T[$. For a process $\mathcal{Y}$ with regulated trajectories, we denote

- $\mathcal{Y}_{t-}=\lim _{s \nearrow t} \mathcal{Y}_{s}$ the left-hand limit of $\mathcal{Y}$ at $\left.\left.t \in\right] 0, T\right],\left(\mathcal{Y}_{0-}=\mathcal{Y}_{0}\right), \mathcal{Y}_{-}:=$ $\left(\mathcal{Y}_{t-}\right)_{t \leq T}$ and $\Delta \mathcal{Y}_{t}:=\mathcal{Y}_{t}-\mathcal{Y}_{t-}$ the size of the left jump of $\mathcal{Y}$ at $t$

- $\mathcal{Y}_{t+}=\lim _{s \searrow t} \mathcal{Y}_{s}$ the right-hand limit of $\mathcal{Y}$ at $t \in\left[0, T\left[,\left(\mathcal{Y}_{T+}=\mathcal{Y}_{T}\right), \mathcal{Y}_{+}:=\right.\right.$ $\left(\mathcal{Y}_{t+}\right)_{t \leq T}$ and $\Delta_{+} \mathcal{Y}_{t}:=\mathcal{Y}_{t+}-\mathcal{Y}_{t}$ the size of the right jump of $\mathcal{Y}$ at $t$.

- For all $t \leq T, \mathcal{Y}_{t}=\mathcal{Y}_{t}^{*}+\sum_{s<t} \Delta_{+} \mathcal{Y}_{s}$ where $\mathcal{Y}^{*}$ is the right-continuous part of the process $\mathcal{Y}$ and $\sum_{s<t} \Delta_{+} \mathcal{Y}_{s}$ stands its purely jumping part consisting of right jumps such that $\sum_{s<t}\left|\Delta_{+} \mathcal{Y}_{s}\right|<+\infty$ a.s.

Let $\xi=\left(\xi_{t}\right)_{t \leq T}$ and $\zeta=\left(\zeta_{t}\right)_{t \leq T}$ be two regulated process such that $\xi_{t} \leq \zeta_{t}$ for all $t \leq T$ a.s. with $\xi_{T}=\zeta_{T}$ a.s. We suppose that $\xi^{+} \in \mathcal{S}_{2 \beta}^{2}$ and $\zeta^{-} \in \mathcal{S}_{2 \beta}^{2}$ where $\xi^{+}=\sup \{\xi, 0\}$ and $\zeta^{-}=\sup \{-\zeta, 0\}$. A pair of process $(\xi, \zeta)$ will be called a pair of irregular barriers.

Definition 2.1. Let $f$ be a stochastic Lipschitz driver and $(\xi, \zeta)$ be a pair of irregular barriers. A process $\left(Y, Z, V, M, K^{+}, K^{-}\right)$is said to be a solution to doubly 
reflected BSDE associated with parameters $(f, \xi, \zeta)$ if

$$
\begin{aligned}
& (i)\left(Y, Z, V, M, K^{+}, K^{-}\right) \in \mathfrak{B}_{\beta}^{2} \times \mathcal{H}_{\beta}^{2} \times \mathcal{L}_{\beta}^{2} \times \mathcal{M}_{\beta}^{2} \times \mathcal{S}^{2} \times \mathcal{S}^{2}, \\
& (i i) Y_{t}=\xi_{T}+\int_{t}^{T} f\left(s, Y_{s}, Z_{s}, V_{s}\right) d s+\left(K_{T}^{+}-K_{t}^{+}\right)-\left(K_{T}^{-}-K_{t}^{-}\right)-\int_{t}^{T} Z_{s} d B_{s} \\
& \quad-\int_{t}^{T} \int_{\mathcal{U}} V_{s}(e) \tilde{\mu}(d s, d e)-\int_{t}^{T} d M_{s}, \\
& \left(\text { iii } \xi_{t} \leq Y_{t} \leq \zeta_{t} \quad \forall t \leq T \text { a.s. },\right.
\end{aligned}
$$$$
\text { (iv)Skorokhod conditions: }\left\{\begin{array}{l}
\int_{0}^{T}\left(Y_{t-}-\xi_{t-}\right) d K_{t}^{+, *}+\sum_{t<T}\left(Y_{t}-\xi_{t}\right) \Delta_{+} K_{t}^{+}=0 \text { a.s. } \\
\int_{0}^{T}\left(\zeta_{t-}-Y_{t-}\right) d K_{t}^{-, *}+\sum_{t<T}\left(\zeta_{t}-Y_{t}\right) \Delta_{+} K_{t}^{-}=0 \text { a.s. }
\end{array}\right.
$$

\section{Existence and uniqueness of solution to DRBSDEs with irregular bar- riers}

The proof of existence and uniqueness result to DRBSDEs with two irregular barriers $(\xi, \zeta)$ will be proved in two steps. Firstly, we consider the case where the driver $f$ does not depend on $(y, z, v)$ and we prove the corresponding result by means a modified penalization method. Secondly, by using fixed point theorem, we prove the main result.

3.1. Penalization method for DRBSDEs with coefficient independent of the solution. Let $f(t, y, z, v):=g(t)$ with $\frac{g}{a} \in \mathcal{H}_{\beta}^{2}$. In what follows, we assume that the irregular barriers $\xi$ and $\zeta$ are strictly separated processes, i.e.

$$
\xi_{t}<\zeta_{t}, \quad \xi_{t-}<\zeta_{t-} \text { and } \xi_{t+}<\zeta_{t+} .
$$

Remark 3.1. The strictly separated assumption on the barriers can be strengthen by the existence of a semimartingale

$$
R_{t}=R_{0}+\int_{0}^{t} \mathcal{Z}_{s} d B_{s}+\int_{0}^{t} \int_{\mathcal{U}} \mathcal{V}_{s}(e) \tilde{\mu}(d s, d e)+\int_{0}^{t} d \mathcal{M}_{s}-J_{t}^{+}+J_{t}^{-}, R_{T}=\xi_{T},
$$

with $(\mathcal{Z}, \mathcal{V}, \mathcal{M}) \in \mathcal{H}^{2} \times \mathcal{L}^{2} \times \mathcal{M}^{2}$ and $J^{ \pm}\left(J_{0}^{ \pm}=0\right)$ are two nondecreasing processes satisfying $\mathbb{E}\left|J_{T}^{ \pm}\right|^{2}<+\infty$ such that

$$
\xi_{t} \leq R_{t} \leq \zeta_{t} \quad 0 \leq t \leq T
$$

By inspiring on Klimsiak et al. (2020) (section 4.2), we consider approximation of the solution to DRBSDE associated with parameters $(g, \xi, \zeta)$ by a modified penalization scheme of the following version

$$
\begin{aligned}
Y_{t}^{n}= & \xi_{T}+\int_{t}^{T} g(s) d s-\int_{t}^{T} Z_{s}^{n} d B_{s}-\int_{t}^{T} \int_{\mathcal{U}} V_{s}^{n}(e) \tilde{\mu}(d s, d e)-\int_{t}^{T} d M_{s}^{n} \\
& +n \int_{t}^{T}\left(Y_{s}^{n}-\xi_{s}\right)^{-} d s+\sum_{t \leq \sigma_{n, i}<T}\left(Y_{\sigma_{n, i}+}^{n}-\xi_{\sigma_{n, i}}\right)^{-} \\
& -n \int_{t}^{T}\left(Y_{s}^{n}-\zeta_{s}\right)^{+} d s-\sum_{t \leq \rho_{n, i}<T}\left(Y_{\rho_{n, i}+}^{n}-\zeta_{\rho_{n, i}}\right)^{+}
\end{aligned}
$$


where $\left\{\sigma_{n, i}\right\}$ and $\left\{\rho_{n, i}\right\}$ are arrays of stopping times exhausting right-jumps of $\xi$ and $\zeta$ respectively defined, for each $n \in \mathbb{N}$, inductively by:

$$
\begin{cases}\sigma_{n, 0}=0 & \\ \sigma_{1, i}=\inf \left\{t>\sigma_{1, i-1} \mid \Delta_{+} \xi_{t}<-1\right\} \wedge T, & i=1, \ldots, k_{1} \\ \sigma_{n+1, i}=\inf \left\{t>\sigma_{n+1, i-1} \mid \Delta_{+} \xi_{t}<-\frac{1}{n+1}\right\} \wedge T, & i=1, \ldots, j_{n+1}\end{cases}
$$

with $k_{1} \in \mathbb{N}$ and $j_{n+1}$ is chosen so that $\mathbb{P}\left(\sigma_{n+1, j_{n+1}}<T\right) \rightarrow 0$ as $n \rightarrow+\infty$ and

$$
\sigma_{n+1, i}=\sigma_{n+1, j_{n+1}} \vee \sigma_{n, i-j_{n+1}}, \quad i=j_{n+1}+1, \ldots, k_{n+1}, \quad k_{n+1}=j_{n+1}+k_{n},
$$

and

$$
\begin{cases}\rho_{n, 0}=0, & \\ \rho_{1, i}=\inf \left\{t>\rho_{1, i-1} \mid \Delta_{+} \zeta_{t}>1\right\} \wedge T, & i=1, \ldots, k_{1} \\ \rho_{n+1, i}=\inf \left\{t>\rho_{n+1, i-1} \mid \Delta_{+} \zeta_{t}>\frac{1}{n+1}\right\} \wedge T, & i=1, \ldots, j_{n+1}\end{cases}
$$

with $k_{1} \in \mathbb{N}$ and $j_{n+1}$ is chosen so that $\mathbb{P}\left(\rho_{n+1, j_{n+1}}<T\right) \rightarrow 0$ as $n \rightarrow+\infty$ and

$$
\rho_{n+1, i}=\rho_{n+1, j_{n+1}} \vee \rho_{n, i-j_{n+1}}, \quad i=j_{n+1}+1, \ldots, k_{n+1}, \quad k_{n+1}=j_{n+1}+k_{n} .
$$

We put, for each $n \in \mathbb{N}$

$$
\left\{\begin{array}{l}
\nu_{n, 0}=0 \\
\nu_{n, 1}=\sigma_{n, 1} \wedge \rho_{n, 1}, \\
\nu_{n, m}=\tilde{\sigma}_{n, m} \wedge \tilde{\rho}_{n, m}, \quad m=2, \ldots, 2 k_{n}
\end{array}\right.
$$

where

$$
\tilde{\sigma}_{n, m}=\min \left\{\sigma_{n, i} \text { such that } \sigma_{n, i}>\nu_{n, m-1} ; \quad i=1, \ldots, k_{n}\right\} \wedge T
$$

and

$$
\tilde{\rho}_{n, m}=\min \left\{\rho_{n, i} \text { such that } \rho_{n, i}>\nu_{n, m-1} ; \quad i=1, \ldots, k_{n}\right\} \wedge T .
$$

Now, to solve the BSDE (3.1), we divide the interval $[0, T]$ into the finite number of intervals $\left.\left.\left[0, \nu_{n, 1}\right], \ldots,\right] \nu_{n, 2 k_{n}-1}, \nu_{n, 2 k_{n}}\right]$ with $\nu_{n, 2 k_{n}}=T$. More precisely, for $m=$ $1, \ldots, 2 k_{n}$, on each interval $\left.] \nu_{n, m-1}, \nu_{n, m}\right]$, the BSDE (3.1) becomes

$$
\begin{array}{r}
Y_{t}^{n}=\xi_{\nu_{n, m}} \vee Y_{\nu_{n, m}}^{n} \wedge \zeta_{\nu_{n, m}}+\int_{t}^{\nu_{n, m}}\left[g(s)+n\left(Y_{s}^{n}-\xi_{s}\right)^{-}-n\left(Y_{s}^{n}-\zeta_{s}\right)^{+}\right] d s \\
\left.\left.-\int_{t}^{\nu_{n, m}} Z_{s}^{n} d B_{s}-\int_{t}^{\nu_{n, m}} \int_{\mathcal{U}} V_{s}^{n}(e) \tilde{\mu}(d s, d e)-\int_{t}^{\nu_{n, m}} d M_{s}^{n}, \quad t \in\right] \nu_{n, m-1}, \nu_{n, m}\right]
\end{array}
$$

with the convention $Y_{T}^{n}=\xi_{T}$ and $Y_{0}^{n}=\xi_{0} \vee Y_{0+}^{n} \wedge \zeta_{0}$. Let $g_{n}(t, y):=g(t)+n(y-$ $\left.\xi_{t}\right)^{-}-n\left(y-\zeta_{t}\right)^{+}$. It's clear that $g_{n}$ is Lipschitz and

$$
\begin{aligned}
& \mathbb{E} \int_{0}^{T} e^{\beta A_{t}}\left|\frac{g^{n}(t, 0)}{a_{t}}\right|^{2} d t
\end{aligned}
$$

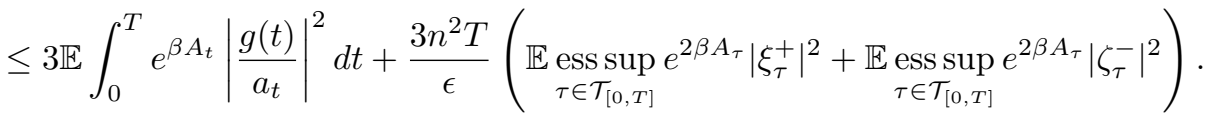

Then, from Theorem A.1 (see Appendix), there exists a unique process $\left(Y^{n}, Z^{n}, V^{n}, M^{n}\right) \in \mathfrak{B}_{\beta}^{2} \times \mathcal{H}_{\beta}^{2} \times \mathcal{L}_{\beta}^{2} \times \mathcal{M}_{\beta}^{2}$ solution of the BSDE (3.2). By induction, 
the $\operatorname{BSDE}(3.1)$ admits a unique solution $\left(Y^{n}, Z^{n}, V^{n}, M^{n}\right) \in \mathfrak{B}_{\beta}^{2} \times \mathcal{H}_{\beta}^{2} \times \mathcal{L}_{\beta}^{2} \times \mathcal{M}_{\beta}^{2}$ and it can be rewritten as

$$
\begin{aligned}
Y_{t}^{n}= & \xi_{T}+\int_{t}^{T} g(s) d s+K_{T}^{+, n}-K_{t}^{+, n}-K_{T}^{-, n}+K_{t}^{-, n}-\int_{t}^{T} Z_{s}^{n} d B_{s} \\
& -\int_{t}^{T} \int_{\mathcal{U}} V_{s}^{n}(e) \tilde{\mu}(d s, d e)-\int_{t}^{T} d M_{s}^{n},
\end{aligned}
$$

where

$$
K_{t}^{+, n}:=K_{t}^{+, n, *}+\sum_{s<t} \Delta_{+} K_{s}^{+, n}=n \int_{0}^{t}\left(Y_{s}^{n}-\xi_{s}\right)^{-} d s+\sum_{0 \leq \sigma_{n, i}<t}\left(Y_{\sigma_{n, i}+}^{n}-\xi_{\sigma_{n, i}}\right)^{-}
$$

and

$$
K_{t}^{-, n}:=K_{t}^{-, n, *}+\sum_{s<t} \Delta_{+} K_{s}^{-, n}=n \int_{0}^{t}\left(Y_{s}^{n}-\zeta_{s}\right)^{+} d s+\sum_{0 \leq \rho_{n, i}<t}\left(Y_{\rho_{n, i}+}^{n}-\zeta_{\rho_{n, i}}\right)^{+} .
$$

Proposition 3.2. Let $\left(Y^{n}, Z^{n}, V^{n}, M^{n}\right) \in \mathfrak{B}_{\beta}^{2} \times \mathcal{H}_{\beta}^{2} \times \mathcal{L}_{\beta}^{2} \times \mathcal{M}_{\beta}^{2}$ for each $n \in \mathbb{N}$, then

$$
\left(\int_{0}^{t} e^{\beta A_{s}}\left\{Y_{s-}^{n} Z_{s}^{n} d B_{s}+\int_{\mathcal{U}} Y_{s-}^{n} V_{s}^{n}(e) \tilde{\mu}(d s, d e)+Y_{s-}^{n} d M_{s}^{n}\right\}\right)_{t \leq T}
$$

is a martingale.

Proof: Let's use the left continuity of trajectories of the process $Y_{s-}^{n}$, we have

$$
\left|Y_{s-}^{n}(\omega)\right|^{2} \leq \sup _{t \in[0, T] \cap \mathbb{Q}}\left|Y_{t-}^{n}(\omega)\right|^{2} \quad \forall(s, \omega) \in[0, T] \times \Omega .
$$

On the other hand, we have $\left|Y_{t-}^{n}\right|^{2} \leq \underset{\tau \in \mathcal{T}_{[0, T]}}{\operatorname{ess} \sup }\left|Y_{\tau}^{n}\right|^{2}$ which implies that

$$
\sup _{t \in[0, T] \cap \mathbb{Q}}\left|Y_{t-}^{n}\right|^{2} \leq \operatorname{esssup}_{\tau \in \mathcal{T}_{[0, T]}}\left|Y_{\tau}^{n}\right|^{2} .
$$

Then for all $\nu \leq \tau \leq T$

$$
\begin{aligned}
\int_{\nu}^{\tau} e^{2 \beta A_{s}}\left|Y_{s-}^{n}\right|^{2}\left|Z_{s}^{n}\right|^{2} d s & \leq \int_{0}^{\tau} e^{2 \beta A_{s}} \sup _{t \in[0, T] \cap \mathbb{Q}}\left|Y_{t-}^{n}\right|^{2}\left|Z_{s}^{n}\right|^{2} d s \\
& \leq \int_{0}^{\tau} e^{2 \beta A_{s}} \operatorname{esssup}_{\tau \in \mathcal{T}_{[0, T]}}\left|Y_{\tau}^{n}\right|^{2}\left|Z_{s}^{n}\right|^{2} d s .
\end{aligned}
$$

Further, we have

$$
\int_{0}^{\tau} e^{2 \beta A_{s}} \underset{\tau \in \mathcal{T}_{[0, T]}}{\operatorname{essups}}\left|Y_{\tau}^{n}\right|^{2}\left|Z_{s}^{n}\right|^{2} d s \leq \underset{\tau \in \mathcal{T}_{[0, T]}}{\operatorname{ess} \sup } e^{\beta A_{\tau}}\left|Y_{\tau}^{n}\right|^{2} \int_{0}^{\tau} e^{\beta A_{s}}\left|Z_{s}^{n}\right|^{2} d s .
$$

Hence

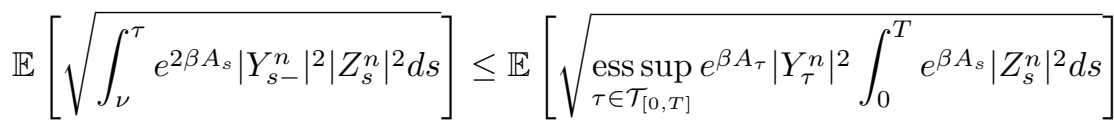

$$
\begin{aligned}
& \leq \frac{1}{2}\left\|Y^{n}\right\|_{\mathcal{S}_{\beta}^{2}}^{2}+\frac{1}{2}\left\|Z^{n}\right\|_{\mathcal{H}_{\beta}^{2}}^{2} .
\end{aligned}
$$

Then the term $\int_{\nu}^{\tau} e^{\beta A_{s}} Y_{s-}^{n} Z_{s}^{n} d B_{s}$ has zero expectation. Since $\left(\int_{0}^{t} e^{\beta A_{s}} Y_{s}^{n} Z_{s}^{n} d B_{s}\right)_{t \leq T}$ is $\mathcal{F}_{t}$-adapted process, then it is a martingale. 
By the same arguments, $\left(\int_{0}^{t} e^{\beta A_{s}}\left\{\int_{\mathcal{U}} Y_{s-}^{n} V_{s}^{n}(e) \tilde{\mu}(d s, d e)+Y_{s-}^{n} d M_{s}^{n}\right\}\right)_{t \leq T}$ is a martingale since

$$
\mathbb{E}\left[\sqrt{\int_{\nu}^{\tau} \int_{\mathcal{U}} e^{2 \beta A_{s}}\left|Y_{s-}^{n}\right|^{2}\left|V_{s}^{n}(e)\right|^{2} \lambda(d e) d s}\right] \leq \frac{1}{2}\left\|Y^{n}\right\|_{\mathcal{S}_{\beta}^{2}}^{2}+\frac{1}{2}\left\|V^{n}\right\|_{\mathcal{L}_{\beta}^{2}}^{2}
$$

and

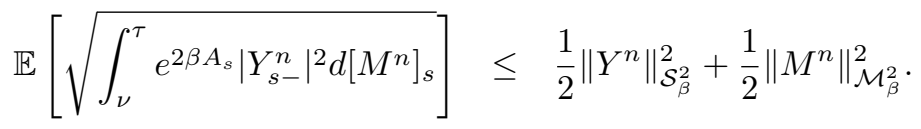

\subsubsection{A priori estimates.}

Lemma 3.3. There exists a positive constant $\kappa$ independent of $n$ such that for all $\beta>1$

$$
\begin{aligned}
& \left\|Y^{n}\right\|_{\mathfrak{B}_{\beta}^{2}}^{2}+\left\|Z^{n}\right\|_{\mathcal{H}_{\beta}^{2}}^{2}+\left\|V^{n}\right\|_{\mathcal{L}_{\beta}^{2}}^{2}+\left\|M^{n}\right\|_{\mathcal{M}_{\beta}^{2}}^{2}+\mathbb{E}\left|K_{T}^{+, n}\right|^{2}+\mathbb{E}\left|K_{T}^{-, n}\right|^{2} \\
& \leq \kappa\left(\left\|\frac{g}{a}\right\|_{\mathcal{H}_{\beta}^{2}}^{2}+\left\|\xi^{+}\right\|_{\mathcal{S}_{2 \beta}^{2}}^{2}+\left\|\zeta^{-}\right\|_{\mathcal{S}_{2 \beta}^{2}}^{2}+\|\mathcal{Z}\|_{\mathcal{H}^{2}}^{2}+\|\mathcal{V}\|_{\mathcal{L}^{2}}^{2}+\|\mathcal{M}\|_{\mathcal{M}^{2}}^{2}+\mathbb{E}\left|J_{T}^{+}\right|^{2}+\mathbb{E}\left|J_{T}^{-}\right|^{2}\right) .
\end{aligned}
$$

Proof: Consider the RBSDE with jumps associated with $(g, \xi)$, that is

$$
\left\{\begin{aligned}
& \bar{Y}_{t}= \xi_{T}+\int_{t}^{T} g(s) d s+\bar{K}_{T}-\bar{K}_{t}-\int_{t}^{T} \bar{Z}_{s} d B_{s}-\int_{t}^{T} \int_{\mathcal{U}} \bar{V}_{s}(e) \tilde{\mu}(d s, d e) \\
&-\int_{t}^{T} d \bar{M}_{s}, \\
& \bar{Y}_{t} \geq \xi_{t} \quad \forall t \leq T \text { and } \int_{0}^{T}\left(\bar{Y}_{t-}-\xi_{t-}\right) d \bar{K}_{t}^{*}+\sum_{t<T}\left(\bar{Y}_{t}-\xi_{t}\right) \Delta_{+} \bar{K}_{t}=0 \text { a.s. }
\end{aligned}\right.
$$

From Theorem A.3 (see Appendix), there exists a unique process $(\bar{Y}, \bar{Z}, \bar{V}, \bar{M}, \bar{K}) \in$ $\mathfrak{B}_{\beta}^{2} \times \mathcal{H}_{\beta}^{2} \times \mathcal{L}_{\beta}^{2} \times \mathcal{M}_{\beta}^{2} \times \mathcal{S}^{2}$ solution of RBSDE (3.3). We consider the penalization equation associated with the RBSDE (3.3), for $n \in \mathbb{N}$

$$
\begin{aligned}
\bar{Y}_{t}^{n}= & \xi_{T}+\int_{t}^{T} g(s) d s+n \int_{t}^{T}\left(\bar{Y}_{s}^{n}-\xi_{s}\right)^{-} d s+\sum_{t \leq \sigma_{n, i}<T}\left(\bar{Y}_{\sigma_{n, i}+}^{n}-\xi_{\sigma_{n, i}}\right)^{-} \\
& -\int_{t}^{T} \bar{Z}_{s}^{n} d B_{s}-\int_{t}^{T} \int_{\mathcal{U}} \bar{V}_{s}^{n}(e) \tilde{\mu}(d s, d e)-\int_{t}^{T} d \bar{M}_{s}^{n} .
\end{aligned}
$$

The comparison theorem A.2 (see Appendix) implies that $\bar{Y}_{t}^{0} \leq \bar{Y}_{t}^{n} \leq \bar{Y}^{n+1}$ and $Y_{t}^{n} \leq \bar{Y}_{t}^{n}$ for all $t \leq T$. Therefore, for all $t \leq T, \bar{Y}_{t}^{n} \nearrow \bar{Y}_{t}$. Hence $Y_{t}^{n} \leq \bar{Y}_{t}$.

Similarly, from Corollary A.4 (see Appendix), there exists a unique process $(\underline{Y}, \underline{Z}, \underline{V}, \underline{M}, \underline{K}) \in \mathfrak{B}_{\beta}^{2} \times \mathcal{H}_{\beta}^{2} \times \mathcal{L}_{\beta}^{2} \times \mathcal{M}_{\beta}^{2} \times \mathcal{S}^{2}$ solution the RBSDE associated with data $(g, \zeta)$, that is

$$
\left\{\begin{aligned}
\underline{Y}_{t}= & \zeta_{T}+\int_{t}^{T} g(s) d s-\left(\underline{K}_{T}-\underline{K}_{t}\right)-\int_{t}^{T} \underline{Z}_{s} d B_{s}-\int_{t}^{T} \int_{\mathcal{U}} \underline{V}_{s}(e) \tilde{\mu}(d s, d e) \\
& -\int_{t}^{T} d \underline{M}_{s}, \\
\underline{Y}_{t} \leq & \zeta_{t} \quad \forall t \leq T \text { and } \int_{0}^{T}\left(\zeta_{t-}-\underline{Y}_{t-}\right) d \underline{K}_{t}^{*}+\sum_{t<T}\left(\zeta_{t}-\underline{Y}_{t}\right) \Delta_{+} \underline{K}_{t}=0 \text { a.s. }
\end{aligned}\right.
$$


By the penalization equation associated with the RBSDE (3.4)

$$
\begin{aligned}
\underline{Y}_{t}^{n}= & \zeta_{T}+\int_{t}^{T} g(s) d s-n \int_{t}^{T}\left(\underline{Y}_{s}^{n}-\zeta_{s}\right)^{+} d s-\sum_{t \leq \rho_{n, i}<T}\left(\underline{Y}_{\rho_{n, i}+}^{n}-\zeta_{\rho_{n, i}}\right)^{+} \\
& -\int_{t}^{T} \underline{Z}_{s}^{n} d B_{s}-\int_{t}^{T} \int_{\mathcal{U}} \underline{V}_{s}^{n}(e) \tilde{\mu}(d s, d e)-\int_{t}^{T} d \underline{M}_{s}^{n}
\end{aligned}
$$

and the comparison theorem A.2 (see Appendix), we deduce that $Y_{t}^{n} \geq \underline{Y}_{t}$ for all $t \leq T$. Then from (A.3) and (A.8) (see Appendix), we can write

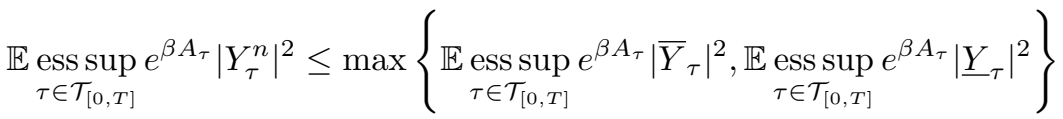

$$
\begin{aligned}
& \leq C\left(2 \mathbb{E} \int_{0}^{T} e^{\beta A_{s}}\left|\frac{g(s)}{a_{s}}\right|^{2} d s+\underset{\tau \in \mathcal{T}_{[0, T]}}{\mathbb{E}} \operatorname{essup}^{2 \beta A_{\tau}}\left(\left|\xi_{\tau}^{+}\right|^{2}+\left|\zeta_{\tau}^{-}\right|^{2}\right)\right)
\end{aligned}
$$

where $C$ is a positive constant. Now, we apply the Corollary A.6 (see Appendix) to $e^{\beta A_{t}}\left|Y_{t}^{n}\right|^{2}$, we have

$$
\begin{aligned}
& e^{\beta A_{t}}\left|Y_{t}^{n}\right|^{2}+\beta \int_{t}^{T} e^{\beta A_{s}}\left|Y_{s}^{n}\right|^{2} d A_{s}+\int_{t}^{T} e^{\beta A_{s}}\left|Z_{s}^{n}\right|^{2} d s+\int_{t}^{T} e^{\beta A_{s}} d\left\langle M^{n}\right\rangle_{s}^{c} \\
= & e^{\beta A_{T}}\left|\xi_{T}\right|^{2}+2 \int_{t}^{T} e^{\beta A_{s}} Y_{s}^{n} g(s) d s-2 \int_{t}^{T} e^{\beta A_{s}} Y_{s}^{n} Z_{s}^{n} d B_{s}-2 \int_{t}^{T} e^{\beta A_{s}} Y_{s-}^{n} d M_{s}^{n} \\
& -2 \int_{t}^{T} \int_{\mathcal{U}} e^{\beta A_{s}} Y_{s-}^{n} V_{s}^{n}(e) \tilde{\mu}(d s, d e)+2 \int_{t}^{T} e^{\beta A_{s}} Y_{s-}^{n}\left(d K_{s}^{+, n, *}-d K_{s}^{-, n, *}\right) \\
& -\sum_{t<s \leq T} e^{\beta A_{s}}\left|\Delta Y_{s}^{n}\right|^{2}-\sum_{t \leq s<T} e^{\beta A_{s}}\left(\left|Y_{s+}^{n}\right|^{2}-\left|Y_{s}^{n}\right|^{2}\right) .
\end{aligned}
$$

Observe that for each $\beta>1$

$$
2 \int_{t}^{T} e^{\beta A_{s}} Y_{s}^{n} g(s) d s \leq(\beta-1) \int_{t}^{T} e^{\beta A_{s}}\left|Y_{s}^{n}\right|^{2} d A_{s}+\frac{1}{\beta-1} \int_{t}^{T} e^{\beta A_{s}}\left|\frac{g(s)}{a_{s}}\right|^{2} d s
$$

and

$$
\left|Y_{s+}^{n}\right|^{2}-\left|Y_{s}^{n}\right|^{2}=\left|\Delta_{+} Y_{s}^{n}\right|^{2}+2 Y_{s}^{n} \Delta_{+} Y_{s}^{n}=\left|\Delta_{+} Y_{s}^{n}\right|^{2}-2 Y_{s}^{n} \Delta_{+}\left(K_{s}^{+, n}-K_{s}^{-, n}\right) .
$$

Recall that $\mu(., d e)$ does not have jumps in common with the processes $K^{ \pm, n}$ for each $n \in \mathbb{N}$, since $\mu(., d e)$ jumps only at totally inaccessible stopping times, then we can note that

$$
\begin{aligned}
& \sum_{t<s \leq T} e^{\beta A_{s}}\left(\Delta Y_{s}^{n}\right)^{2} \\
= & \int_{t}^{T} \int_{\mathcal{U}} e^{\beta A_{s}}\left|V_{s}^{n}(e)\right|^{2} \mu(d s, d e)+\sum_{t<s \leq T} e^{\beta A_{s}}\left(-\Delta K_{s}^{+, n}+\Delta K_{s}^{-, n}+\Delta M_{s}^{n}\right)^{2} .
\end{aligned}
$$


Then, one can derive that

$$
\begin{aligned}
& \int_{t}^{T} e^{\beta A_{s}}\left\|V_{s}^{n}\right\|_{\lambda}^{2} d s+\sum_{t<s \leq T} e^{\beta A_{s}}\left(\Delta M_{s}^{n}\right)^{2}-\sum_{t<s \leq T} e^{\beta A_{s}}\left(\Delta Y_{s}^{n}\right)^{2} \\
= & -\int_{t}^{T} \int_{\mathcal{U}} e^{\beta A_{s}}\left|V_{s}^{n}(e)\right|^{2} \tilde{\mu}(d s, d e)-\sum_{t<s \leq T} e^{\beta A_{s}}\left(\Delta K_{s}^{+, n}-\Delta K_{s}^{-, n}\right)^{2} \\
& -2 \sum_{t<s \leq T} e^{\beta A_{s}}\left(\Delta K_{s}^{+, n}-\Delta K_{s}^{-, n}\right) \Delta M_{s}^{n} \\
\leq & -\int_{t}^{T} \int_{\mathcal{U}} e^{\beta A_{s}}\left|V_{s}^{n}(e)\right|^{2} \tilde{\mu}(d s, d e)-2 \sum_{t<s \leq T} e^{\beta A_{s}}\left(\Delta K_{s}^{+, n}-\Delta K_{s}^{-, n}\right) \Delta M_{s}^{n} .
\end{aligned}
$$

By adding the term $\int_{t}^{T} e^{\beta A_{s}}\left\|V_{s}^{n}\right\|_{\lambda}^{2} d s+\sum_{t<s \leq T} e^{\beta A_{s}}\left(\Delta M_{s}^{n}\right)^{2}$ on both sides of inequality (3.6), by taking in consideration (3.7) and (3.8) and by using the basic equality $\left[M^{n}\right]_{s}=\left\langle M^{n}\right\rangle_{s}^{c}+\sum_{t<s \leq T}\left(\Delta M_{s}^{n}\right)^{2}$, we deduce

$$
\begin{aligned}
& e^{\beta A_{t}}\left|Y_{t}^{n}\right|^{2}+\int_{t}^{T} e^{\beta A_{s}}\left|Y_{s}^{n}\right|^{2} d A_{s}+\int_{t}^{T} e^{\beta A_{s}}\left(\left|Z_{s}^{n}\right|^{2}+\left\|V_{s}^{n}\right\|_{\lambda}^{2}\right) d s+\int_{t}^{T} e^{\beta A_{s}} d\left[M^{n}\right]_{s} \\
& \leq \operatorname{esssup}_{\tau \in \mathcal{T}_{[0, T]}} e^{2 \beta A_{\tau}}\left|\xi_{\tau}^{+}\right|^{2}+\int_{t}^{T} e^{\beta A_{s}}\left|\frac{g(s)}{a_{s}}\right|^{2} d s+2 \int_{t}^{T} e^{\beta A_{s}} Y_{s-}^{n}\left(d K_{s}^{+, n}-d K_{s}^{-, n}\right) \\
& -2 \int_{t}^{T} e^{\beta A_{s}} Y_{s}^{n} Z_{s}^{n} d B_{s}-\int_{t}^{T} \int_{\mathcal{U}} e^{\beta A_{s}}\left(2 Y_{s-}^{n} V_{s}^{n}(e)+\left|V_{s}^{n}(e)\right|^{2}\right) \tilde{\mu}(d s, d e) \\
& -2 \int_{t}^{T} e^{\beta A_{s}} Y_{s-}^{n} d M_{s}^{n}-2 \sum_{t<s \leq T} e^{\beta A_{s}}\left(\Delta K_{s}^{+, n}-\Delta K_{s}^{-, n}\right) \Delta M_{s}^{n} .
\end{aligned}
$$

Taking expectation on the both sides of the inequality (3.9) for $t=0$ and using the Proposition 3.2, we get

$$
\begin{aligned}
& \mathbb{E} \int_{0}^{T} e^{\beta A_{s}}\left|Y_{s}^{n}\right|^{2} d A_{s}+\mathbb{E} \int_{0}^{T} e^{\beta A_{s}}\left(\left|Z_{s}^{n}\right|^{2}+\left\|V_{s}^{n}\right\|_{\lambda}^{2}\right) d s+\mathbb{E} \int_{0}^{T} e^{\beta A_{s}} d\left[M^{n}\right]_{s}
\end{aligned}
$$

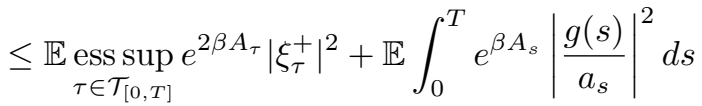

$$
\begin{aligned}
& +2 \mathbb{E} \int_{t}^{T} e^{\beta A_{s}} Y_{s-}^{n}\left(d K_{s}^{+, n}-d K_{s}^{-, n}\right)-2 \mathbb{E} \sum_{t<s \leq T} e^{\beta A_{s}}\left(\Delta K_{s}^{+, n}-\Delta K_{s}^{-, n}\right) \Delta M_{s}^{n} .
\end{aligned}
$$

Since $M^{n}$ is a martingale then for each predictable stopping time $\tau \in \mathcal{T}_{[0, T]}$ $\mathbb{E}\left[\Delta M_{\tau}^{n} \mid \mathcal{F}_{\tau-}\right]=0$ a.s. Moreover, since the processes $K^{+, n}$ and $K^{-, n}$ are pre-

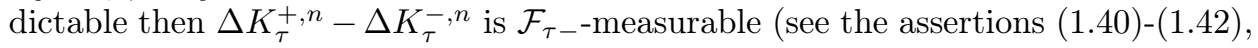
Chapter I in Jacod, 1979). Therefore,

$$
\mathbb{E}\left[\left(\Delta K_{\tau}^{+, n}-\Delta K_{\tau}^{-, n}\right) \Delta M_{\tau}^{n} \mid \mathcal{F}_{\tau-}\right]=\left(\Delta K_{\tau}^{+, n}-\Delta K_{\tau}^{-, n}\right) \mathbb{E}\left[\Delta M_{\tau}^{n} \mid \mathcal{F}_{\tau-}\right]=0
$$

Hence the term $\sum_{t<s \leq T} e^{\beta A_{s}}\left(\Delta K_{s}^{+, n}-\Delta K_{s}^{-, n}\right) \Delta M_{s}^{n}$ has a zero expectation. Now, let us come back to the expression (3.2), for each $t \in\left[\nu_{n, m-1}, \nu_{n, m}\right.$ [ it holds true 
that

$$
\begin{aligned}
\int_{0}^{t} e^{\beta A_{s}} Y_{s-}^{n} d K_{s}^{+, n} & =\int_{0}^{t} e^{\beta A_{s}} Y_{s}^{n} n\left(Y_{s}^{n}-\xi_{s}\right)^{-} d s \\
& =\int_{0}^{t} e^{\beta A_{s}} \xi_{s} n\left(Y_{s}^{n}-\xi_{s}\right)^{-} d s-\int_{0}^{t} e^{\beta A_{s}} n\left(\left(Y_{s}^{n}-\xi_{s}\right)^{-}\right)^{2} d s \\
& \leq \int_{0}^{t} e^{\beta A_{s}} \xi_{s}^{+} n\left(Y_{s}^{n}-\xi_{s}\right)^{-} d s=\int_{0}^{t} e^{\beta A_{s}} \xi_{s}^{+} d K_{s}^{+, n},
\end{aligned}
$$

and similarly $-\int_{0}^{t} e^{\beta A_{s}} Y_{s}^{n} d K_{s}^{-, n} \leq \int_{0}^{t} e^{\beta A_{s}} \zeta_{s}^{-} d K_{s}^{-, n}$. Consequently, for some $\alpha_{2}>0$

$$
\begin{aligned}
& \mathbb{E} \int_{0}^{T} e^{\beta A_{s}}\left|Y_{s}^{n}\right|^{2} d A_{s}+\mathbb{E} \int_{0}^{T} e^{\beta A_{s}}\left(\left|Z_{s}^{n}\right|^{2}+\left\|V_{s}^{n}\right\|_{\lambda}^{2}\right) d s+\mathbb{E} \int_{0}^{T} e^{\beta A_{s}} d\left[M^{n}\right]_{s}
\end{aligned}
$$

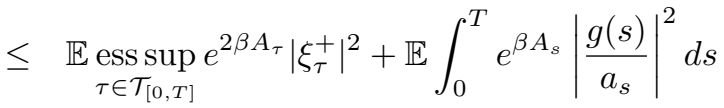

$$
\begin{aligned}
& +\alpha_{2} \mathbb{E} \underset{\tau \in \mathcal{T}_{[0, T]}}{\operatorname{ess} \sup } e^{2 \beta A_{\tau}}\left(\left|\xi_{\tau}^{+}\right|^{2}+\left|\zeta_{\tau}^{-}\right|^{2}\right)+\frac{1}{\alpha_{2}} \mathbb{E}\left|K_{T}^{+, n}\right|^{2}+\frac{1}{\alpha_{2}} \mathbb{E}\left|K_{T}^{-, n}\right|^{2} .
\end{aligned}
$$

To conclude, we now give an estimate of $\mathbb{E}\left|K_{T}^{+, n}\right|^{2}$ and $\mathbb{E}\left|K_{T}^{-, n}\right|^{2}$. Let us introduce the following stopping times for each $n \in \mathbb{N}^{*}$

$$
\begin{cases}\tau_{n, 0}=0 \\ \tau_{n, 2 i+1}=\inf \left\{t>\tau_{n, 2 i} \mid Y_{t}^{n}=\xi_{t}\right\} \wedge T, & i \geq 0 \\ \tau_{n, 2 i+2}=\inf \left\{t>\tau_{n, 2 i+1} \mid Y_{t}^{n}=\zeta_{t}\right\} \wedge T, & i \geq 0\end{cases}
$$

Since $\xi<\zeta$, then $\tau_{n, i}<\tau_{n, i+1}$ on the set $\left\{\tau_{n, i+1}<T\right\}$. In addition the sequence $\left(\tau_{n, i}\right)_{i \geq 0}$ is of stationary type (i.e. $\forall \omega \in \Omega$, there exists $i_{0}(\omega)$ such that $\forall i \geq i_{0}(\omega)$, $\left.\tau_{n, i}(\omega)=\tau_{n, i+1}(\omega)=T\right)$. Indeed, let us set $\mathcal{G}=\bigcap_{i \geq 0}\left\{\tau_{n, i}<T\right\}$, we show that $\mathbb{P}(\mathcal{G})=0$. We assume that $\mathbb{P}(\mathcal{G})>0$, therefore for $\omega \in \mathcal{G}$, there exists two sequences of real numbers $\left(k_{n, i}(\omega)\right)_{i \geq 0}$ and $\left(k_{n, i}^{\prime}(\omega)\right)_{i \geq 0}$ belongs to $\left[\tau_{n, i-1}, \tau_{n, i}\right]$ such that

and

$$
\left\{\begin{array}{l}
Y_{k_{n, i}}^{n}=\zeta_{k_{n, i}} \wedge \zeta_{k_{n, i}-}=\zeta_{k_{n, i}}-\left(\Delta \zeta_{k_{n, i}}\right)^{+} \\
Y_{k_{n, i}}^{n}=\zeta_{k_{n, i}} \wedge \zeta_{k_{n, i}+}=\zeta_{k_{n, i}}-\left(\Delta_{+} \zeta_{k_{n, i}}\right)^{-}
\end{array}\right.
$$

$$
\left\{\begin{array}{c}
Y_{k_{n, i}^{\prime}}^{n}=\xi_{k_{n, i}^{\prime}} \vee \xi_{k_{n, i}^{\prime}-}=\xi_{k_{n, i}^{\prime}}-\left(\Delta \xi_{k_{n, i}^{\prime}}\right)^{-} \\
Y_{k_{n, i}^{\prime}}^{n}=\xi_{k_{n, i}^{\prime}} \vee \xi_{k_{n, i}^{\prime}}^{\prime}=\xi_{k_{n, i}^{\prime}}-\left(\Delta_{+} \xi_{k_{n, i}^{\prime}}\right)^{+}
\end{array}\right.
$$

Now as $\left(k_{n, i}\right)_{i \geq 0}$ and $\left(k_{n, i}^{\prime}\right)_{i \geq 0}$ are not of stationary type since $\left(\tau_{n, i}\right)_{i \geq 0}$ is nondecreasing sequence then taking the limit as $i \rightarrow+\infty$ to obtain that $Y_{\tau-}^{n}(\omega)=$ $\xi_{\tau-}(\omega) \leq \zeta_{\tau-}(\omega)=Y_{\tau-}^{n}(\omega)$ and $Y_{\tau+}^{n}(\omega)=\xi_{\tau+}(\omega) \leq \zeta_{\tau+}(\omega)=Y_{\tau+}^{n}(\omega)$. Then $\xi_{\tau-}(\omega)=\zeta_{\tau-}(\omega)$ and $\xi_{\tau+}(\omega)=\zeta_{\tau+}(\omega)$, but this is contradiction since $\mathbb{P}$-a.s. $\forall t \leq T, \xi_{t-}<\zeta_{t-}$ and $\xi_{t+}<\zeta_{t+}$. We deduce that $\mathbb{P}(\mathcal{G})=0$.

Next, let $p \geq 1$ be a real number, and $\varsigma_{\tau_{n, 2 i}}^{p}$ and $\varsigma_{\tau_{n, 2 i+1}}^{p}$ be a stopping times defined by:

and

$$
\varsigma_{\tau_{n, 2 i}}^{p}=\inf \left\{t>\tau_{n, 2 i} \mid Y_{t}^{n} \leq \xi_{t}+\frac{1}{p}\right\} \wedge T
$$

$$
\varsigma_{\tau_{n, 2 i+1}}^{p}=\inf \left\{t>\tau_{n, 2 i+1} \mid Y_{t}^{n} \geq \zeta_{t}-\frac{1}{p}\right\} \wedge T \text {. }
$$


Then for all $t \in\left[\tau_{n, 2 i}, \varsigma_{\tau_{n, 2 i}}^{p}\right]$, we have $Y_{t-}^{n}>\xi_{t-}$ and for all $t \in\left[\tau_{n, 2 i+1}, \varsigma_{\tau_{n, 2 i+1}}^{p}\right]$, we have $Y_{t-}^{n}<\zeta_{t-}$. Consequently, it holds true that $Y^{n}>\xi$ on $\left[\tau_{n, 2 i}, \tau_{n, 2 i+1} \wedge \varsigma_{\tau_{n, 2 i}}^{p}\right]$ and $Y^{n}<\zeta$ on $\left[\tau_{n, 2 i+1}, \tau_{n, 2 i+2} \wedge \varsigma_{\tau_{n, 2 i+1}}^{p}\right]$. It follows the BSDE (3.1) becomes

$$
\begin{aligned}
Y_{\tau_{n, 2 i}}^{n}= & Y_{\tau_{n, 2 i+1} \wedge \varsigma_{\tau_{n, 2 i}}^{p}}^{n}+\int_{\tau_{n, 2 i}}^{\tau_{n, 2 i+1} \wedge \varsigma_{\tau_{n, 2 i}}^{p}} g(s) d s-\int_{\tau_{n, 2 i}}^{\tau_{n, 2 i+1} \wedge \varsigma_{\tau_{n, 2 i}}^{p}} Z_{s}^{n} d B_{s} \\
& -\int_{\tau_{n, 2 i}}^{\tau_{n, 2 i+1} \wedge \varsigma_{\tau_{n, 2 i}}^{p}} \int_{\mathcal{U}} V_{s}^{n}(e) \tilde{\mu}(d s, d e)-\int_{\tau_{n, 2 i}}^{\tau_{n, 2 i+1} \wedge \varsigma_{\tau_{n, 2 i}}^{p}} d M_{s}^{n} \\
& -n \int_{\tau_{n, 2 i}}^{\tau_{n, 2 i+1} \wedge \varsigma_{\tau_{n, 2 i}}^{p}}\left(Y_{s}^{n}-\zeta_{s}\right)^{+} d s-\sum_{\tau_{n, 2 i} \leq \rho_{n, i}<\tau_{n, 2 i+1} \wedge \varsigma_{\tau_{n, 2 i}}^{p}}\left(Y_{\rho_{n, i}+}^{n}-\zeta_{\rho_{n, i}}\right)^{+}
\end{aligned}
$$

On the other hand, by Remark 3.1, we have $Y_{\tau_{n, 2 i}}^{n}>R_{\tau_{n, 2 i}}$ on $\left\{\tau_{n, 2 i}<T\right\}, Y_{\tau_{n, 2 i}}^{n}=$ $R_{\tau_{n, 2 i}}=\xi_{T}=\zeta_{T}$ on $\left\{\tau_{n, 2 i}=T\right\}, Y_{\tau_{n, 2 i+1} \wedge \varsigma_{\tau_{n, 2 i}}^{p}}^{n} \leq R_{\tau_{n, 2 i+1} \wedge \varsigma_{\tau_{n, 2 i}}^{p}}+\frac{1}{p}$ on $\left\{\tau_{n, 2 i+1} \wedge\right.$ $\left.\varsigma_{\tau_{n, 2 i}}^{p}<T\right\}$ and $Y_{\tau_{n, 2 i+1} \wedge \varsigma_{\tau_{n, 2 i}}^{p}}^{n}=R_{\tau_{n, 2 i+1} \wedge \varsigma_{\tau_{n, 2 i}}^{p}}=\xi_{T}=\zeta_{T}$ on $\left\{\tau_{n, 2 i+1} \wedge \varsigma_{\tau_{n, 2 i}}^{p}=T\right\}$. From (3.11) and definition of the process $R$ we obtain

$$
\begin{aligned}
& n \int_{\tau_{n, 2 i}}^{\tau_{n, 2 i+1} \wedge \varsigma_{\tau_{n, 2 i}}^{p}}\left(Y_{s}^{n}-\zeta_{s}\right)^{+} d s+\sum_{\tau_{n, 2 i} \leq \rho_{n, i}<\tau_{n, 2 i+1} \wedge \varsigma_{\tau_{n, 2 i}}^{p}}\left(Y_{\rho_{n, i}+}^{n}-\zeta_{\rho_{n, i}}\right)^{+} \\
= & Y_{\tau_{n, 2 i+1} \wedge \varsigma_{\tau_{n, 2 i}}^{p}}^{n}-Y_{\tau_{n, 2 i}}^{n}+\int_{\tau_{n, 2 i}}^{\tau_{n, 2 i+1} \wedge \varsigma_{\tau_{n, 2 i}^{p}}^{p}} g(s) d s-\int_{\tau_{n, 2 i}}^{\tau_{n, 2 i+1} \wedge \varsigma_{\tau_{n, 2 i}}^{p}} Z_{s}^{n} d B_{s} \\
& -\int_{\tau_{n, 2 i}}^{\tau_{n, 2 i+1} \wedge \varsigma_{\tau_{n, 2 i}}^{p}} d M_{s}^{n}-\int_{\tau_{n, 2 i}}^{\tau_{n, 2 i+1} \wedge \varsigma_{\tau_{n, 2 i}}^{p}} \int_{\mathcal{U}} V_{s}^{n}(e) \tilde{\mu}(d s, d e) \\
\leq & R_{\tau_{n, 2 i+1} \wedge \varsigma_{\tau_{n, 2 i}}^{p}}+\frac{1}{p}-R_{\tau_{n, 2 i}}+\int_{\tau_{n, 2 i}}^{\tau_{n, 2 i+1} \wedge \varsigma_{\tau_{n, 2 i}}^{p}}|g(s)| d s-\int_{\tau_{n, 2 i}}^{\tau_{n, 2 i+1} \wedge \varsigma_{\tau_{n, 2 i}}^{p}} Z_{s}^{n} d B_{s} \\
& -\int_{\tau_{n, 2 i}}^{\tau_{n, 2 i+1} \wedge \varsigma_{\tau_{n, 2 i}}^{p}} d M_{s}^{n}-\int_{\tau_{n, 2 i}}^{\tau_{n, 2 i+1} \wedge \varsigma_{\tau_{n, 2 i}}^{p}} \int_{\mathcal{U}} V_{s}^{n}(e) \tilde{\mu}(d s, d e) \\
\leq & \frac{1}{p}+\int_{\tau_{n, 2 i}}^{\tau_{n, 2 i+1} \wedge \varsigma_{\tau_{n, 2 i}}^{p}}|g(s)| d s+\int_{\tau_{n, 2 i}}^{\tau_{n, 2 i+1} \wedge \varsigma_{\tau_{n, 2 i}}^{p}}\left(\mathcal{Z}_{s}-Z_{s}^{n}\right) d B_{s} \\
& +\int_{\tau_{n, 2 i}}^{\tau_{n, 2 i+1} \wedge \varsigma_{\tau_{n, 2 i}}^{p}} d\left(\mathcal{M}_{s}-M_{s}^{n}\right)+\int_{\tau_{n, 2 i}}^{\tau_{n, 2 i+1} \wedge \varsigma_{\tau_{n, 2 i}}^{p}} \int_{\mathcal{U}}\left(\mathcal{V}_{s}(e)-V_{s}^{n}(e)\right) \tilde{\mu}(d s, d e) \\
& +J_{\tau_{n, 2 i+1} \wedge \varsigma_{\tau_{n, 2 i}}^{p}}^{-}-J_{\tau_{n, 2 i}}^{-} .
\end{aligned}
$$

Taking the limit as $p \rightarrow+\infty$, using the fact that $Y^{n}<\zeta$ on the interval $\left[\tau_{n, 2 i+1}, \tau_{n, 2 i+2} \wedge \varsigma_{\tau_{n, 2 i+1}}^{p}\right]$, summing in $i$ and taking expectation in the both sides of 
above inequality, we obtain

$$
\begin{aligned}
& \mathbb{E}\left[n \int_{t}^{T}\left(Y_{s}^{n}-\zeta_{s}\right)^{+} d s+\sum_{t \leq \rho_{n, i}<T}\left(Y_{\rho_{n, i}+}^{n}-\zeta_{\rho_{n, i}}\right)^{+}\right]^{2} \\
\leq & 8\left(\frac{1}{\beta} \mathbb{E} \int_{0}^{T}\left|\frac{g(s)}{a_{s}}\right|^{2} d s+\mathbb{E} \int_{0}^{T}\left(\left|\mathcal{Z}_{s}\right|^{2}+\left\|\mathcal{V}_{s}\right\|_{\lambda}^{2}\right) d s+\mathbb{E} \int_{0}^{T} d[\mathcal{M}]_{s}\right. \\
& \left.+\mathbb{E} \int_{0}^{T} e^{\beta A_{s}}\left(\left|Z_{s}^{n}\right|^{2}+\left\|V_{s}^{n}\right\|_{\lambda}^{2}\right) d s+\mathbb{E} \int_{0}^{T} e^{\beta A_{s}} d\left[M^{n}\right]_{s}+\mathbb{E}\left|J_{T}^{-}\right|^{2}\right) .
\end{aligned}
$$

In the same way, we can obtain

$$
\begin{aligned}
& \mathbb{E}\left[n \int_{t}^{T}\left(Y_{s}^{n}-\xi_{s}\right)^{-} d s+\sum_{t \leq \sigma_{n, i}<T}\left(Y_{\sigma_{n, i}+}^{n}-\xi_{\sigma_{n, i}}\right)^{-}\right]^{2} \\
\leq & 8\left(\frac{1}{\beta} \mathbb{E} \int_{0}^{T}\left|\frac{g(s)}{a_{s}}\right|^{2} d s+\mathbb{E} \int_{0}^{T}\left(\left|\mathcal{Z}_{s}\right|^{2}+\left\|\mathcal{V}_{s}\right\|_{\lambda}^{2}\right) d s+\mathbb{E} \int_{0}^{T} d[\mathcal{M}]_{s}\right. \\
+ & \left.\mathbb{E} \int_{0}^{T} e^{\beta A_{s}}\left(\left|Z_{s}^{n}\right|^{2}+\left\|V_{s}^{n}\right\|_{\lambda}^{2}\right) d s+\mathbb{E} \int_{0}^{T} e^{\beta A_{s}} d\left[M^{n}\right]_{s}+\mathbb{E}\left|J_{T}^{+}\right|^{2}\right) .
\end{aligned}
$$

The desired result obtained by combining (3.12), (3.13) with (3.10) for $\alpha_{2}>8 c$, and adding the estimate (3.5).

\subsubsection{Existence of (limiting) solution to DRBSDEs.}

Lemma 3.4. There exists a quadruple of processes $(Y, Z, V, M)$ such that

$$
\left\|Y^{n}-Y\right\|_{\mathfrak{B}_{\beta}^{2}}^{2}+\left\|Z^{n}-Z\right\|_{\mathcal{H}_{\beta}^{2}}^{2}+\left\|V^{n}-V\right\|_{\mathcal{L}_{\beta}^{2}}^{2}+\left\|M^{n}-M\right\|_{\mathcal{M}_{\beta}^{2}}^{2} \underset{n \rightarrow+\infty}{\longrightarrow} 0 .
$$

Proof: For each $n>p \geq 0$, we apply the Corollary A.6 (see Appendix) to get

$$
\begin{aligned}
& e^{\beta A_{t}}\left|Y_{t}^{n}-Y_{t}^{p}\right|^{2}+\beta \int_{t}^{T} e^{\beta A_{s}}\left|Y_{s}^{n}-Y_{s}^{p}\right|^{2} d A_{s}+\int_{t}^{T} e^{\beta A_{s}}\left|Z_{s}^{n}-Z_{s}^{p}\right|^{2} d s \\
& +\int_{t}^{T} e^{\beta A_{s}} d\left\langle M^{n}-M^{p}\right\rangle_{s}^{c} \\
= & -2 \int_{t}^{T} e^{\beta A_{s}}\left(Y_{s}^{n}-Y_{s}^{p}\right)\left(Z_{s}^{n}-Z_{s}^{p}\right) d B_{s}-2 \int_{t}^{T} e^{\beta A_{s}}\left(Y_{s-}^{n}-Y_{s-}^{p}\right) d\left(M_{s}^{n}-M_{s}^{p}\right) \\
& -2 \int_{t}^{T} \int_{\mathcal{U}} e^{\beta A_{s}}\left(Y_{s-}^{n}-Y_{s-}^{p}\right)\left(V_{s}^{n}(e)-V_{s}^{p}(e)\right) \tilde{\mu}(d s, d e) \\
& +2 \int_{t}^{T} e^{\beta A_{s}}\left(Y_{s-}^{n}-Y_{s-}^{p}\right) d\left[\left(K_{s}^{+, n, *}-K_{s}^{+, p, *}\right)-\left(K_{s}^{-, n, *}-K_{s}^{-, p, *}\right)\right] \\
& -\sum_{t<s \leq T} e^{\beta A_{s}}\left|\Delta\left(Y_{s}^{n}-Y_{s}^{p}\right)\right|^{2}-\sum_{t \leq s<T} e^{\beta A_{s}}\left(\left|Y_{s+}^{n}-Y_{s+}^{p}\right|^{2}-\left|Y_{s}^{n}-Y_{s}^{p}\right|^{2}\right)(3.14)
\end{aligned}
$$


By using the same computations as those used to get (3.9), and the fact that

$$
\begin{aligned}
& \left|Y_{s+}^{n}-Y_{s+}^{p}\right|^{2}-\left|Y_{s}^{n}-Y_{s}^{p}\right|^{2} \\
= & \left|\Delta_{+}\left(Y_{s}^{n}-Y_{s}^{p}\right)\right|^{2}+2\left(Y_{s}^{n}-Y_{s}^{p}\right) \Delta_{+}\left(Y_{s}^{n}-Y_{s}^{p}\right) \\
= & \left|\Delta_{+}\left(Y_{s}^{n}-Y_{s}^{p}\right)\right|^{2}-2\left(Y_{s}^{n}-Y_{s}^{p}\right) \Delta_{+}\left[\left(K_{s}^{+, n}-K_{s}^{+, p}\right)-\left(K_{s}^{-, n}-K_{s}^{-, p}\right)\right],
\end{aligned}
$$

we can derive that

$$
\begin{aligned}
& \beta \mathbb{E} \int_{0}^{T} e^{\beta A_{s}}\left|Y_{s}^{n}-Y_{s}^{p}\right|^{2} d A_{s}+\mathbb{E} \int_{0}^{T} e^{\beta A_{s}}\left(\left|Z_{s}^{n}-Z_{s}^{p}\right|^{2}+\left\|V_{s}^{n}-V_{s}^{p}\right\|_{\lambda}^{2}\right) d s \\
& +\mathbb{E} \int_{0}^{T} e^{\beta A_{s}} d\left[M^{n}-M^{p}\right]_{s} \\
\leq & 2 \mathbb{E} \int_{0}^{T} e^{\beta A_{s}}\left(Y_{s-}^{n}-Y_{s-}^{p}\right) d\left[\left(K_{s}^{+, n}-K_{s}^{+, p}\right)-\left(K_{s}^{-, n}-K_{s}^{-, p}\right)\right] \\
\leq & 2 \mathbb{E}\left[\sup _{0 \leq t \leq T} e^{\beta A_{t}}\left(Y_{t-}^{p}-\xi_{t-}\right)^{-} K_{T}^{+, n}\right]+2 \mathbb{E}\left[\sup _{0 \leq t \leq T} e^{\beta A_{t}}\left(Y_{t-}^{n}-\xi_{t-}\right)^{-} K_{T}^{+, p}\right] \\
& +2 \mathbb{E}\left[\sup _{0 \leq t \leq T} e^{\beta A_{t}}\left(Y_{t-}^{p}-\zeta_{t-}\right)^{+} K_{T}^{-, n}\right]+2 \mathbb{E}\left[\sup _{0 \leq t \leq T} e^{\beta A_{t}}\left(Y_{t-}^{n}-\zeta_{t-}\right)^{+} K_{T}^{-, p}\right] .
\end{aligned}
$$

Then, for $\beta>1$

$$
\begin{aligned}
& \left\|Y^{n}-Y^{p}\right\|_{\mathcal{S}_{\beta}^{2, A}}^{2}+\left\|Z^{n}-Z^{p}\right\|_{\mathcal{H}_{\beta}^{2}}^{2}+\left\|V^{n}-V^{p}\right\|_{\mathcal{L}_{\beta}^{2}}^{2}+\left\|M^{n}-M^{p}\right\|_{\mathcal{M}_{\beta}^{2}}^{2} \\
\leq & 2 \mathbb{E}\left[\sup _{0 \leq t \leq T} e^{2 \beta A_{t}}\left|\left(Y_{t}^{p}-\xi_{t}\right)^{-}\right|^{2}\right]^{\frac{1}{2}}\left(\mathbb{E}\left|K_{T}^{+, n}\right|^{2}\right)^{\frac{1}{2}} \\
+ & 2 \mathbb{E}\left[\sup _{0 \leq t \leq T} e^{2 \beta A_{t}}\left|\left(Y_{t}^{n}-\xi_{t}\right)^{-}\right|^{2}\right]^{\frac{1}{2}}\left(\mathbb{E}\left|K_{T}^{+, p}\right|^{2}\right)^{\frac{1}{2}} \\
+ & 2 \mathbb{E}\left[\sup _{0 \leq t \leq T} e^{2 \beta A_{t}}\left|\left(Y_{t}^{p}-\zeta_{t}\right)^{+}\right|^{2}\right]^{\frac{1}{2}}\left(\mathbb{E}\left|K_{T}^{-, n}\right|^{2}\right)^{\frac{1}{2}} \\
+ & 2 \mathbb{E}\left[\sup _{0 \leq t \leq T} e^{2 \beta A_{t}}\left|\left(Y_{t}^{n}-\zeta_{t}\right)^{+}\right|^{2}\right]^{\frac{1}{2}}\left(\mathbb{E}\left|K_{T}^{-, p}\right|^{2}\right)^{\frac{1}{2}}
\end{aligned}
$$

It remains to prove that

$$
\mathbb{E}\left[\sup _{0 \leq t \leq T} e^{2 \beta A_{t}}\left|\left(Y_{t}^{n}-\xi_{t}\right)^{-}\right|^{2}+\sup _{0 \leq t \leq T} e^{2 \beta A_{t}}\left|\left(Y_{t}^{n}-\zeta_{t}\right)^{+}\right|^{2}\right] \underset{n \rightarrow+\infty}{\longrightarrow} 0 .
$$

Indeed, from Theorem A.1 (see Appendix), there exists a unique process $\left(\widehat{Y}^{n}, \widehat{Z}^{n}, \widehat{V}^{n}, \widehat{M}^{n}\right)$ solution to the following BSDE

$$
\begin{aligned}
\widehat{Y}_{t}^{n}= & \xi_{T}+\int_{t}^{T}\left[g(s)+n\left(\xi_{s}-\widehat{Y}_{s}^{n}\right)\right] d s-\int_{t}^{T} \widehat{Z}_{s}^{n} d B_{s}-\int_{t}^{T} \int_{\mathcal{U}} \widehat{V}_{s}^{n}(e) \tilde{\mu}(d s, d e) \\
& -\int_{t}^{T} d \widehat{M}_{s}^{n} .
\end{aligned}
$$

Since $\left(\xi_{s}-\widehat{Y}_{s}^{n}\right)=\left(\widehat{Y}_{s}^{n}-\xi_{s}\right)^{-}-\left(\xi_{s}-\widehat{Y}_{s}^{n}\right)^{-}$then Theorem A.2 (see Appendix) implies $Y_{t}^{n} \geq \widehat{Y}_{t}^{n}$ for all $t \leq T$. For any stopping time $\nu \leq T$ we have

$$
\widehat{Y}_{\nu}^{n}=\mathbb{E}\left[e^{-n(T-\nu)} \xi_{T}+\int_{\nu}^{T} e^{-n(s-\nu)} g(s) d s+n \int_{\nu}^{T} e^{-n(s-\nu)} \xi_{s} d s \mid \mathcal{F}_{\nu}\right]
$$


But

$$
e^{-n(T-\nu)} \xi_{T}+n \int_{\nu}^{T} e^{-n(s-\nu)} \xi_{s} d s \underset{n \rightarrow+\infty}{\longrightarrow} \xi_{\nu} \mathbb{1}_{\nu \leq T} \quad \mathbb{P}-\text { a.s. and in } \mathcal{L}^{2} .
$$

Moreover, the conditional expectation converges also in $\mathcal{L}^{2}$. In addition, by Hölder inequality, we have

$$
\left|\int_{\nu}^{T} e^{-n(s-\nu)} g(s) d s\right|^{2} \leq\left(\int_{\nu}^{T} e^{\beta A_{s}}\left|\frac{g(s)}{a_{s}}\right|^{2} d s\right)\left(\int_{\nu}^{T} e^{-2 n(s-\nu)-\beta A_{s}} d A_{s}\right) .
$$

Thus $\mathbb{E}\left[\int_{\nu}^{T} e^{-n(s-\nu)} g(s) d s \mid \mathcal{F}_{\nu}\right] \underset{n \rightarrow+\infty}{\longrightarrow} 0 \mathbb{P}-$ a.s. Now, we denote

$$
\widehat{y}_{t}^{n}:=e^{-n(T-t)} \xi_{T}+\int_{t}^{T} e^{-n(s-t)}\left(g(s)+n \xi_{s}\right) d s
$$

From (3.17), Jensen's inequality and Doob's maximal quadratic inequality (see theorem 20, p. 11 in Protter, 2005), we have

$$
\begin{aligned}
\mathbb{E}\left[\sup _{0 \leq t \leq T} e^{2 \beta A_{t}}\left|\left(\widehat{Y}_{t}^{n}-\xi_{t}\right)^{-}\right|^{2}\right] & =\mathbb{E}\left[\sup _{0 \leq t \leq T} e^{2 \beta A_{t}}\left|\mathbb{E}\left[\widehat{y}_{t}^{n}-\xi_{t} \mid \mathcal{F}_{t}\right]^{-}\right|^{2}\right] \\
& \leq \mathbb{E}\left[\sup _{0 \leq t \leq T}\left|\mathbb{E}\left[e^{\beta A_{t}}\left(\widehat{y}_{t}^{n}-\xi_{t}\right)^{-} \mid \mathcal{F}_{t}\right]\right|^{2}\right] \\
& \leq 4 \mathbb{E}\left[\left|e^{\beta A_{t}}\left(\widehat{y}_{t}^{n}-\xi_{t}\right)^{-}\right|^{2}\right] \\
& \leq 4 \mathbb{E}\left[\sup _{0 \leq t \leq T} e^{2 \beta A_{t}}\left|\left(\widehat{y}_{t}^{n}-\xi_{t}\right)^{-}\right|^{2}\right] .
\end{aligned}
$$

On the other hand, the sequence define as

$$
\left(X_{t}^{n}\right)_{n \geq 1}:=\left\{e^{-n(T-t)} \xi_{T}+n \int_{t}^{T} e^{-n(s-t)} \xi_{s} d s-\xi_{t}\right\}_{n \geq 1}
$$

is uniform converge in $t$ and also for $\left(e^{\beta A_{t}}\left(X_{t}^{n}\right)^{-}\right)_{n \geq 1}$. Lebesgue's dominated convergence theorem implies that

$$
\begin{aligned}
& \lim _{n \rightarrow+\infty} \mathbb{E}\left[\sup _{0 \leq t \leq T} e^{2 \beta A_{t}}\left|\left(\widehat{y}_{t}^{n}-\xi_{t}\right)^{-}\right|^{2}\right] \\
\leq & 2 \lim _{n \rightarrow+\infty} \mathbb{E}\left[\sup _{0 \leq t \leq T} e^{2 \beta A_{t}}\left|X_{t}^{n-}\right|^{2}+\sup _{0 \leq t \leq T} e^{2 \beta A_{t}}\left|\int_{t}^{T} e^{-n(s-t)} g(s) d s\right|^{2}\right]=0 .
\end{aligned}
$$

Since $Y_{t}^{n} \geq \widehat{Y}_{t}^{n} \forall t \leq T$, we deduce $\mathbb{E}\left[\sup _{0 \leq t \leq T} e^{2 \beta A_{t}}\left|\left(Y_{t}^{n}-\xi_{t}\right)^{-}\right|^{2}\right] \underset{n \rightarrow+\infty}{\longrightarrow} 0$.

Similarly we can obtain $\mathbb{E}\left[\sup _{0 \leq t \leq T} e^{2 \beta A_{t}}\left|\left(Y_{t}^{n}-\zeta_{t}\right)^{+}\right|^{2}\right] \underset{n \rightarrow+\infty}{\longrightarrow} 0$.

Now, passing to the limit in (3.15), we obtain

$$
\left\|Y^{n}-Y^{p}\right\|_{\mathcal{S}_{\beta}^{2, A}}^{2}+\left\|Z^{n}-Z^{p}\right\|_{\mathcal{H}_{\beta}^{2}}^{2}+\left\|V^{n}-V^{p}\right\|_{\mathcal{L}_{\beta}^{2}}^{2}+\left\|M^{n}-M^{p}\right\|_{\mathcal{M}_{\beta}^{2}}^{2} \underset{n, p \rightarrow+\infty}{\longrightarrow} 0
$$


which implies that $\left(Y^{n}, Z^{n}, V^{n}, M^{n}\right)_{n \geq 0}$ is a Cauchy sequence in $\mathcal{S}_{\beta}^{2, A} \times \mathcal{H}_{\beta}^{2} \times \mathcal{L}_{\beta}^{2} \times$ $\mathcal{M}_{\beta}^{2}$. So there exists a quadruple $(Y, Z, V, M) \in \mathcal{S}_{\beta}^{2, A} \times \mathcal{H}_{\beta}^{2} \times \mathcal{L}_{\beta}^{2} \times \mathcal{M}_{\beta}^{2}$ such that

$$
\left\|Y^{n}-Y\right\|_{\mathcal{S}_{\beta}^{2, A}}^{2}+\left\|Z^{n}-Z\right\|_{\mathcal{H}_{\beta}^{2}}^{2}+\left\|V^{n}-V\right\|_{\mathcal{L}_{\beta}^{2}}^{2}+\left\|M^{n}-M\right\|_{\mathcal{M}_{\beta}^{2}}^{2} \underset{n \rightarrow+\infty}{\longrightarrow} 0 .
$$

On the other hand, from Remark A.1 in Grigorova et al. (2017), ess $\sup _{\tau \in \mathcal{T}_{[0, T]}} X_{\tau}=$ $\sup _{t \leq T} X_{t}$ for all càdlàg process $X$, then by Burkholder-Davis-Gundy's inequality, there exists a universal positive constant $c$ such that

$$
\begin{aligned}
& 2 \mathbb{E} \underset{\tau \in \mathcal{T}_{[0, T]} \operatorname{ess} \sup _{0}}{ }\left|\int_{0}^{\tau} e^{\beta A_{s}}\left(Y_{s}^{n}-Y_{s}^{p}\right)\left(Z_{s}^{n}-Z_{s}^{p}\right) d B_{s}\right| \\
& \leq 2 c \mathbb{E}\left[\sqrt{\int_{0}^{T} e^{2 \beta A_{s}}\left|Y_{s}^{n}-Y_{s}^{p}\right|^{2}\left|Z_{s}^{n}-Z_{s}^{p}\right|^{2} d s}\right] \\
& \leq \frac{1}{4} \mathbb{E} \underset{\tau \in \mathcal{T}_{[0, T]}}{\operatorname{ess} \sup } e^{\beta A_{\tau}}\left|Y_{\tau}^{n}-Y_{\tau}^{p}\right|^{2}+4 c^{2} \mathbb{E} \int_{0}^{T} e^{\beta A_{s}}\left|Z_{s}^{n}-Z_{s}^{p}\right|^{2} d s \\
& =\frac{1}{4}\left\|Y^{n}-Y^{p}\right\|_{\mathcal{S}_{\beta}^{2}}^{2}+4 c^{2}\left\|Z^{n}-Z^{p}\right\|_{\mathcal{H}_{\beta}^{2}}^{2}, \\
& 2 \mathbb{E} \underset{\tau \in \mathcal{T}_{[0, T]}}{\operatorname{ess} \sup }\left|\int_{0}^{\tau} \int_{\mathcal{U}} e^{\beta A_{s}}\left(Y_{s-}^{n}-Y_{s-}^{p}\right)\left(V_{s}^{n}(e)-V_{s}^{p}(e)\right) \tilde{\mu}(d s, d e)\right| \\
& \leq 2 c \mathbb{E}\left[\sqrt{\int_{0}^{T} \int_{\mathcal{U}} e^{2 \beta A_{s}}\left|Y_{s-}^{n}-Y_{s-}^{p}\right|^{2}\left|V_{s}^{n}(e)-V_{s}^{p}(e)\right|^{2} \mu(d s, d e)}\right] \\
& \leq \frac{1}{4} \mathbb{E} \underset{\tau \in \mathcal{T}_{[0, T]}}{\operatorname{essup} e^{\beta A_{\tau}}}\left|Y_{\tau}^{n}-Y_{\tau}^{p}\right|^{2}+4 c^{2} \mathbb{E} \int_{0}^{T} \int_{\mathcal{U}} e^{\beta A_{s}}\left|V_{s}^{n}(e)-V_{s}^{p}(e)\right|^{2} \mu(d s, d e) \\
& =\frac{1}{4}\left\|Y^{n}-Y^{p}\right\|_{\mathcal{S}_{\beta}^{2}}^{2}+4 c^{2}\left\|V^{n}-V^{p}\right\|_{\mathcal{L}_{\beta}^{2}}^{2}
\end{aligned}
$$

and

$$
\begin{aligned}
& 2 \mathbb{E} \underset{\tau \in \mathcal{T}_{[0, T]} \operatorname{ess} \sup _{0}}{ }\left|\int_{0}^{\tau} e^{\beta A_{s}}\left(Y_{s-}^{n}-Y_{s-}^{p}\right) d\left(M_{s}^{n}-M_{s}^{p}\right)\right| \\
& \leq 2 c \mathbb{E}\left[\sqrt{\int_{0}^{T} e^{2 \beta A_{s}}\left|Y_{s-}^{n}-Y_{s-}^{p}\right|^{2} d\left[M^{n}-M^{p}\right]_{s}}\right]
\end{aligned}
$$

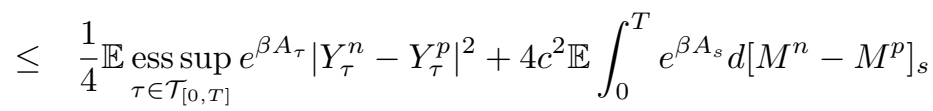

$$
\begin{aligned}
& =\frac{1}{4}\left\|Y^{n}-Y^{p}\right\|_{\mathcal{S}_{\beta}^{2}}^{2}+4 c^{2}\left\|M^{n}-M^{p}\right\|_{\mathcal{M}_{\beta}^{2}}^{2} .
\end{aligned}
$$


Consequently, by taking the essential supremum over $\tau \in \mathcal{T}_{[0, T]}$ and then the expectation on both sides of inequality (3.14) we get

$$
\begin{aligned}
& \left\|Y^{n}-Y^{p}\right\|_{\mathcal{S}_{\beta}^{2}}^{2} \\
\leq & 4\left(4 c^{2}\left\|Z^{n}-Z^{p}\right\|_{\mathcal{H}_{\beta}^{2}}^{2}+4 c^{2}\left\|V^{n}-V^{p}\right\|_{\mathcal{L}_{\beta}^{2}}^{2}+4 c^{2}\left\|M^{n}-M^{p}\right\|_{\mathcal{M}_{\beta}^{2}}^{2}\right. \\
& +2 \mathbb{E}\left[\sup _{0 \leq t \leq T} e^{\beta A_{t}}\left(Y_{t-}^{p}-\xi_{t-}\right)^{-} K_{T}^{+, n}\right]+2 \mathbb{E}\left[\sup _{0 \leq t \leq T} e^{\beta A_{t}}\left(Y_{t-}^{n}-\xi_{t-}\right)^{-} K_{T}^{+, p}\right] \\
& \left.+2 \mathbb{E}\left[\sup _{0 \leq t \leq T} e^{\beta A_{t}}\left(Y_{t-}^{p}-\zeta_{t-}\right)^{+} K_{T}^{-, n}\right]+2 \mathbb{E}\left[\sup _{0 \leq t \leq T} e^{\beta A_{t}}\left(Y_{t-}^{n}-\zeta_{t-}\right)^{+} K_{T}^{-, p}\right]\right) \\
& \underset{n, p \rightarrow+\infty}{\longrightarrow} 0 .
\end{aligned}
$$

Then, $\left\|Y^{n}-Y\right\|_{\mathcal{S}_{\beta}^{2}}^{2} \underset{n \rightarrow+\infty}{\longrightarrow} 0$ and $Y \in \mathcal{S}_{\beta}^{2}$.

Lemma 3.5. There exists two optional processes $K^{+}$and $K^{-}$with left and right finite limits such that

- $\underset{\tau \in \mathcal{T}_{[0, T]}}{\mathbb{E} \operatorname{ess} \sup }\left|K_{\tau}^{+, n}-K_{\tau}^{+}\right|^{2}+\underset{\tau \in \mathcal{T}_{[0, T]}}{\mathbb{E}} \operatorname{ess} \sup \left|K_{\tau}^{-, n}-K_{\tau}^{-}\right|^{2} \underset{n \rightarrow+\infty}{\longrightarrow} 0$

- For all $t \leq T, K_{t}^{+}=K_{t}^{+, *}+\sum_{t<T} \Delta_{+} K_{t}^{+}$with

$$
\int_{0}^{T}\left(Y_{t-}-\xi_{t-}\right) d K_{t}^{+, *}+\sum_{t<T}\left(Y_{t}-\xi_{t}\right) \Delta_{+} K_{t}^{+}=0 \text { a.s. }
$$

- For all $t \leq T, K_{t}^{-}=K_{t}^{-, *}+\sum_{t<T} \Delta_{+} K_{t}^{-}$with

$$
\int_{0}^{T}\left(\zeta_{t-}-Y_{t-}\right) d K_{t}^{-, *}+\sum_{t<T}\left(\zeta_{t}-Y_{t}\right) \Delta_{+} K_{t}^{-}=0 \text { a.s. }
$$

Proof: For each $n \in \mathbb{N}$, we consider the modified penalization BSDE (3.1) which be can take the following form

$$
\left\{\begin{array}{l}
Y_{t}^{n}=\xi_{T}+\int_{t}^{T} g_{n}\left(s, Y_{s}^{n}\right) d s+K_{T}^{+, n}-K_{t}^{+, n}-\int_{t}^{T} Z_{s}^{n} d B_{s}-\int_{t}^{T} \int_{\mathcal{U}} V_{s}^{n}(e) \tilde{\mu}(d s, d e) \\
\quad-\int_{t}^{T} d M_{s}^{n}, \\
Y_{t}^{n} \geq \xi_{t} \quad \forall t \leq T, \\
\int_{0}^{T}\left(Y_{t-}^{n}-\xi_{t-}\right) d K_{t}^{+, n, *}+\sum_{t<T}\left(Y_{t}^{n}-\xi_{t}\right) \Delta_{+} K_{t}^{+, n}=0 \text { a.s. }
\end{array}\right.
$$

where $g_{n}(t, y)=g(t)-n\left(y-\zeta_{t}\right)^{+}-\sum_{t \leq \rho_{n, i}<T}\left(y_{\rho_{n, i}+}-\zeta_{\rho_{n, i}}\right)^{+}$. Since $g_{n+1}(t, y)$ $\leq g_{n}(t, y)$, then from Remark 4.3, $K_{t}^{+, n} \leq K_{t}^{+, n+1}$, for all $t \leq T$. Therefore, there exists an optional process $K^{+}$such that $K^{+, n} \nearrow K^{+}$. Using the fact that

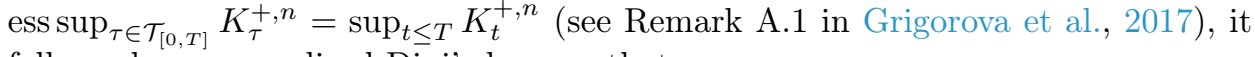
follows, by a generalized Dini's lemma, that

$$
\mathbb{E}\left[\operatorname{ess~sup}_{\tau \in \mathcal{T}_{[0, T]}}\left|K_{\tau}^{+, n}-K_{\tau}^{+}\right|^{2}\right] \underset{n \rightarrow+\infty}{\longrightarrow} 0 .
$$


On the other hand we have

$K_{t}^{-, n}=Y_{t}^{n}-Y_{0}^{n}+\int_{0}^{t} g(s) d s+K_{t}^{n,+}-\int_{0}^{t} Z_{s}^{n} d B_{s}-\int_{0}^{t} \int_{\mathcal{U}} V_{s}^{n}(e) \tilde{\mu}(d s, d e)-\int_{0}^{t} d M_{s}^{n}$.

Then, from Burkholder-Davis-Gundy's inequality there exists a universal constant $c$ such that, for each $n \geq p>0$

$$
\begin{aligned}
& \mathbb{E} \underset{\tau \in \mathcal{T}_{[0, T]}}{\operatorname{ess} \sup }\left|K_{\tau}^{-, n}-K_{\tau}^{-, p}\right|^{2} \\
& \leq 6\left(\underset{\tau \in \mathcal{T}_{[0, T]}}{\mathbb{E} \operatorname{ess} \sup }\left|Y_{\tau}^{n}-Y_{\tau}^{p}\right|^{2}+\mathbb{E}\left|Y_{0}^{n}-Y_{0}^{p}\right|^{2}+\underset{\tau \in \mathcal{T}_{[0, T]}}{\mathbb{E}} \operatorname{essup}\left|K_{\tau}^{+, n}-K_{\tau}^{+, p}\right|^{2}\right. \\
& +c \mathbb{E} \int_{0}^{T} e^{\beta A_{s}}\left\{\left|Z_{s}^{n}-Z_{s}^{p}\right|^{2}+\left\|V_{s}^{n}-V_{s}^{p}\right\|_{\lambda}^{2}\right\} d s \\
& \left.+c \mathbb{E} \int_{0}^{T} e^{\beta A_{s}} d\left[M^{n}-M^{p}\right]_{s}\right) \underset{n, p \rightarrow+\infty}{\longrightarrow} 0 .
\end{aligned}
$$

Consequently, there exists an optional process $K^{-}$such that

$$
\mathbb{E}\left[\operatorname{ess~sup~}_{\tau \in \mathcal{T}_{[0, T]}}\left|K_{\tau}^{-, n}-K_{\tau}^{-}\right|^{2}\right] \underset{n \rightarrow+\infty}{\longrightarrow} 0 .
$$

In what follows, we are going to show that the Skorokhod condition for the optional processes $K^{+}$and $K^{-}$is satisfied. We know that

$$
\int_{0}^{T}\left(\zeta_{t-}-Y_{t-}^{n}\right) d K_{t}^{n,-, *}+\sum_{t<T}\left(\zeta_{t-}-Y_{t}^{n}\right) \Delta_{+} K_{t}^{-, n}=0 \text { a.s. }
$$

Notice that $d K_{t}^{-, n} \nearrow d K_{t}^{-}$in the total variation norm (to be precise $\Delta_{+} K_{t}^{-, n} \nearrow$ $\Delta_{+} K_{t}^{-}$and $d K_{t}^{-, n, *} \nearrow d K_{t}^{-, *}$ in the total variation norm). Since $0 \leq \zeta_{t-}-Y_{t}^{n} \leq$ $\zeta_{t-}-Y_{t}^{0}$. By using the Lebesgue dominated convergence theorem we get

$$
\int_{0}^{T}\left(\zeta_{t-}-Y_{t-}\right) d K_{t}^{-, *}+\sum_{t<T}\left(\zeta_{t-}-Y_{t}\right) \Delta_{+} K_{t}^{-}=0 \text { a.s. }
$$

Further, by the integrability properties of $Y$ and $K^{-}$, the process $\left(Y_{t}+\int_{0}^{t} g(s) d s+\right.$ $\left.K_{t}^{-}\right)_{t \leq T}$ is a supermartingale which dominates the process $\left(\xi_{t}+\int_{0}^{t} g(s) d s+K_{t}^{-}\right)_{t \leq T}$. Hence

$$
Y_{t} \geq \underset{\tau \in \mathcal{T}_{[0, T]}}{\operatorname{ess} \sup } \mathbb{E}\left[\xi_{t}+\int_{t}^{\tau} g(s) d s-K_{\tau}^{-}+K_{t}^{-} \mid \mathcal{F}_{t}\right] .
$$

On the other hand, choosing an optimal stopping time in order to get the reversed inequality, let $\delta_{t}=\inf \left\{t \leq s \leq T, Y_{s}=\xi_{s}\right\}$ with $\delta_{T}=T \mathbb{1}_{\{Y>\xi\}}$. Then

$$
Y_{t}=\operatorname{ess~sup}_{\delta_{t} \in \mathcal{T}_{[0, T]}} \mathbb{E}\left[\xi_{\delta_{t}} \mathbb{1}_{\delta_{t} \leq T}+\int_{t}^{\delta_{t}} g(s) d s-K_{\delta_{t}}^{-}+K_{t}^{-} \mid \mathcal{F}_{t}\right] .
$$

The Skorokhod condition (3.19) implies that $K_{\delta_{t}}^{-}-K_{t}^{-}=0$. Then, $Y$ is the Snell envelope of the optional process $\xi$. The process $K^{+}$, obtained in (3.18), 
coincides with the increasing process from Mertens decomposition of $Y$, therefore, from Corollary 3.11 in Klimsiak et al. (2019) we can write

$$
\int_{0}^{T}\left(Y_{t-}-\xi_{t-}\right) d K_{t}^{+, *}+\sum_{t<T}\left(Y_{t}-\xi_{t}\right) \Delta_{+} K_{t}^{+}=0 \text { a.s. }
$$

Theorem 3.6. The limit $\left(Y, Z, V, M, K^{+}, K^{-}\right)$of $\left(Y^{n}, Z^{n}, V^{n}, M^{n}, K^{+, n}, K^{-, n}\right)$ is the unique solution of the DRBSDE associated with parameters $(g, \xi, \zeta)$.

Proof: By combining the lemmas 3.4 and 3.5, passing to limit as $n \longrightarrow+\infty$ in

$$
\begin{aligned}
Y_{t}^{n}= & \xi_{T}+\int_{t}^{T} g(s) d s+\left(K_{T}^{+, n}-K_{t}^{+, n}\right)-\left(K_{T}^{-, n}-K_{t}^{-, n}\right)-\int_{t}^{T} Z_{s}^{n} d B_{s} \\
& -\int_{t}^{T} \int_{\mathcal{U}} V_{s}^{n}(e) \tilde{\mu}(d s, d e)-\int_{t}^{T} d M_{s}^{n}
\end{aligned}
$$

to obtain

$$
\begin{aligned}
Y_{t}= & \xi_{T}+\int_{t}^{T} g(s) d s+\left(K_{T}^{+}-K_{t}^{+}\right)-\left(K_{T}^{-}-K_{t}^{-}\right)-\int_{t}^{T} Z_{s} d B_{s} \\
& -\int_{t}^{T} \int_{\mathcal{U}} V_{s}(e) \tilde{\mu}(d s, d e)-\int_{t}^{T} d M_{s}
\end{aligned}
$$

with the Skorokhod's conditions for $K^{+}$and $K^{-}$are satisfied. Moreover, since the sequence $\left(Y^{n}, Z^{n}, V^{n}, M^{n}, K^{+, n}, K^{-, n}\right)$ is belong to the Banach space $\mathfrak{B}_{\beta}^{2} \times \mathcal{H}_{\beta}^{2} \times$ $\mathcal{L}_{\beta}^{2} \times \mathcal{M}_{\beta}^{2} \times \mathcal{S}^{2} \times \mathcal{S}^{2}$, then the limit $\left(Y, Z, V, M, K^{+}, K^{-}\right)$stay in same space. To conclude, from (3.16) we get $\xi_{t} \leq Y_{t} \leq \zeta_{t}$ for all $t \leq T$ a.s.

\subsection{The solution of DRBSDEs with general coefficient.}

Theorem 3.7. Let $f$ be a stochastic Lipschitz driver and $(\xi, \zeta)$ be a pair of irregular barriers. The DRBSDE with jumps associated with parameters $(f, \xi, \zeta)$ has a unique solution $\left(Y, Z, V, M, K^{+}, K^{-}\right)$which belongs to $\mathfrak{B}_{\beta}^{2} \times \mathcal{H}_{\beta}^{2} \times \mathcal{L}_{\beta}^{2} \times \mathcal{M}_{\beta}^{2} \times \mathcal{S}^{2} \times \mathcal{S}^{2}$ for each $\beta>4$.

Proof: Let $\mathcal{D}_{\beta}=\mathcal{S}_{\beta}^{2, A} \times \mathcal{H}_{\beta}^{2} \times \times \mathcal{L}_{\beta}^{2}$. We define a mapping $\Phi$ from $\mathcal{D}_{\beta}$ into itself as follows: Given $(y, z, v) \in \mathcal{D}_{\beta}$ and obviously that

$$
\mathbb{E} \int_{0}^{T} e^{\beta A_{s}}\left|\frac{f\left(s, y_{s}, z_{s}, v_{s}\right)}{a_{s}}\right|^{2} d s<+\infty .
$$

Then from Theorem 3.6, there exists a unique $\left(Y, Z, V, K^{+}, K^{-}\right)$solution of the DRBSDE associated with parameters $(f(., y, z, v), \xi, \zeta)$, then we put $\Phi(y, z, v)=$ $(Y, Z, V)$. Let us show that $\Phi$ is a contraction and hence admits a unique fixed point $(Y, Z, V) \in \mathcal{D}_{\beta}$, which corresponds to the unique solution of DRBSDE associated with parameters $(f, \xi, \zeta)$. Let $\left(y^{\prime}, z^{\prime}, v^{\prime}\right)$ be another element of $\mathcal{D}_{\beta}$ and define $\Phi\left(y^{\prime}, z^{\prime}, v^{\prime}\right)=\left(Y^{\prime}, Z^{\prime}, V^{\prime}\right)$ where the process $\left(Y^{\prime}, Z^{\prime}, V^{\prime}, M^{\prime}, K^{\prime+}, K^{\prime-}\right)$ is the unique solution of the DRBSDE associated with parameters $\left(f\left(., y^{\prime}, z^{\prime}, v^{\prime}\right), \xi, \zeta\right)$. Set $\bar{\Re}=$ $\Re-\Re^{\prime}$ for $\Re \in\left\{Y, Z, V, M, K^{ \pm}, y, z, v\right\}$, and we put $\bar{f}_{t}=f\left(t, y_{t}, z_{t}, v_{t}\right)-f\left(t, y_{t}^{\prime}, z_{t}^{\prime}, v_{t}^{\prime}\right)$ 
for all $t \leq T$. Using Corollary A.6 (see Appendix) and some standard computations, we get

$$
\begin{aligned}
& \beta \mathbb{E} \int_{t}^{T} e^{\beta A_{s}}\left|\bar{Y}_{s}\right|^{2} d A_{s}+\mathbb{E} \int_{t}^{T} e^{\beta A_{s}}\left(\left|\bar{Z}_{s}\right|^{2}+\left\|\bar{V}_{s}\right\|_{\lambda}^{2}\right) d s+\mathbb{E} \int_{t}^{T} e^{\beta A_{s}} d[\bar{M}, \bar{M}]_{s} \\
\leq & 2 \mathbb{E} \int_{t}^{T} e^{\beta A_{s}} \bar{Y}_{s} \bar{f}_{s} d s+2 \mathbb{E} \int_{t}^{T} e^{\beta A_{s}} \bar{Y}_{s-}\left(d \bar{K}_{s}^{+, *}-d \bar{K}_{s}^{-, *}\right) \\
& +2 \mathbb{E} \sum_{t \leq s<T} e^{\beta A_{s}} \bar{Y}_{s}\left(\Delta_{+} \bar{K}_{s}^{+}-\Delta_{+} \bar{K}_{s}^{-}\right) .
\end{aligned}
$$

Thanks to the Skorokhod conditions on $K^{+}$and $K^{-}$, we have $\int_{t}^{T} e^{\beta A_{s}} \bar{Y}_{s-}\left(d \bar{K}_{s}^{+, *}-\right.$ $\left.d \bar{K}_{s}^{-, *}\right) \leq 0$ and

$$
\begin{aligned}
& \sum_{t \leq s<T} e^{\beta A_{s}} \bar{Y}_{s}\left(\Delta_{+} \bar{K}_{s}^{+}-\Delta_{+} \bar{K}_{s}^{-}\right) \\
= & \sum_{t \leq s<T} e^{\beta A_{s}}\left(Y_{s}-\xi_{s}\right) \Delta_{+} K_{s}^{+}-\sum_{t \leq s<T} e^{\beta A_{s}}\left(Y_{s}^{\prime}-\xi_{s}\right) \Delta_{+} K_{s}^{+} \\
& +\sum_{t \leq s<T} e^{\beta A_{s}}\left(Y_{s}^{\prime}-\xi_{s}\right) \Delta_{+} K_{s}^{\prime+}-\sum_{t \leq s<T} e^{\beta A_{s}}\left(Y_{s}-\xi_{s}\right) \Delta_{+} K_{s}^{\prime+} \\
& -\sum_{t \leq s<T} e^{\beta A_{s}}\left(Y_{s}-\zeta_{s}\right) \Delta_{+} K_{s}^{-}+\sum_{t \leq s<T} e^{\beta A_{s}}\left(Y_{s}^{\prime}-\zeta_{s}\right) \Delta_{+} K_{s}^{-} \\
& -\sum_{t \leq s<T} e^{\beta A_{s}}\left(Y_{s}^{\prime}-\zeta_{s}\right) \Delta_{+} K_{s}^{\prime}+\sum_{t \leq s<T} e^{\beta A_{s}}\left(Y_{s}-\zeta_{s}\right) \Delta_{+} K_{s}^{\prime} \\
\leq & 0 .
\end{aligned}
$$

Moreover, by using the stochastic Lipschitz condition on $f$, we get for any $\beta>4$

$$
\begin{aligned}
& \mathbb{E} \int_{t}^{T} e^{\beta A_{s}}\left|\bar{Y}_{s}\right|^{2} d A_{s}+\mathbb{E} \int_{t}^{T} e^{\beta A_{s}}\left(\left|\bar{Z}_{s}\right|^{2}+\left\|\bar{V}_{s}\right\|_{\lambda}^{2}\right) d s+\mathbb{E} \int_{t}^{T} e^{\beta A_{s}} d[\bar{M}, \bar{M}]_{s} \\
\leq & \frac{3}{\beta-1}\left(\mathbb{E} \int_{t}^{T} e^{\beta A_{s}}\left|\bar{y}_{s}\right|^{2} d A_{s}+\mathbb{E} \int_{t}^{T} e^{\beta A_{s}}\left|\bar{z}_{s}\right|^{2} d s+\mathbb{E} \int_{t}^{T} e^{\beta A_{s}}\left\|\bar{v}_{s}\right\|_{\lambda}^{2} d s\right) .
\end{aligned}
$$

Then the mapping $\Phi$ is a contraction and then has a unique fixed point $(Y, Z, V)$ which actually belongs to $\mathcal{D}_{\beta}$. Moreover, there exists $\left(M, K^{+}, K^{-}\right) \in \mathcal{M}_{\beta}^{2} \times \mathcal{S}^{2} \times \mathcal{S}^{2}$ $\left(K_{0}^{ \pm}=0\right)$ such that $\left(Y, Z, V, M, K^{+}, K^{-}\right)$is a unique solution of the DRBSDE associated with $(f, \xi, \zeta)$.

\section{Comparison theorem}

The comparison theorem is one of the principal tools in the theories of the BSDEs. But it does not hold in general for solutions of BSDEs with jumps (see the counter example in Barles et al., 1997). However, it's shown in special cases (see for example Royer, 2006; Yin and Mao, 2008).

In order to obtain the comparison theorem, we will discus the following generator:

$$
f^{1}(\omega, t, y, z, v)=F(\omega, t, y, z)+\int_{\mathcal{U}} \pi_{t}(\omega, e) v(e) \lambda(d e)
$$

where 
- $\pi: \Omega \times[0, T] \times \mathcal{U} \longrightarrow[-1,+\infty[$ is a $\mathcal{P} \otimes$ U-measurable mapping such that $\int_{\mathcal{U}}\left|\pi_{t}(\omega, e)\right|^{2} \lambda(d e)<+\infty$.

- One has $\frac{F(., 0,0)}{a} \in \mathcal{H}_{\beta}^{2}$ and $\forall\left(y, y^{\prime}, z, z^{\prime}\right) \in \mathbb{R}^{4}$

$$
\left|F(\omega, t, y, z)-F\left(\omega, t, y^{\prime}, z^{\prime}\right)\right| \leq \theta_{t}\left|y-y^{\prime}\right|+\gamma_{t}\left|z-z^{\prime}\right|
$$

Let $\left(Y^{j}, Z^{j}, V^{j}, M^{j}, K^{+, j}, K^{-, j}\right)$ be the unique solution of the DRBSDE associated with data $\left(f^{j}, \xi^{j}, \zeta^{j}\right)$, for $j=1,2$. Then we have the following result:

Theorem 4.1. Assume that $f^{1}\left(., Y^{2}, Z^{2}, V^{2}\right) \leq f^{2}\left(., Y^{2}, Z^{2}, V^{2}\right)$ a.s., $\xi^{1} \leq \xi^{2}$ a.s. and $\zeta^{1} \leq \zeta^{2}$ a.s. Then $Y^{1} \leq Y^{2}$ a.s.

Proof: Let us put $\bar{\Re}=\Re^{1}-\Re^{2}$ for $\Re \in\left\{Y, Z, V, M, K^{+}, K^{-}, \xi, \zeta\right\}$. Then

$$
\begin{aligned}
\bar{Y}_{t}= & \bar{\xi}_{T}+\int_{t}^{T}\left(\varphi_{s} \bar{Y}_{s}+\psi_{s} \bar{Z}_{s}+\int_{\mathcal{U}} \pi_{t}(\omega, e) \bar{V}_{s}(e) \lambda(d e)+\phi_{s}\right) d s+\left(\bar{K}_{T}^{+}-\bar{K}_{t}^{+}\right) \\
& -\left(\bar{K}_{T}^{-}-\bar{K}_{t}^{-}\right)-\int_{t}^{T} \bar{Z}_{s} d B_{s}-\int_{t}^{T} \int_{\mathcal{U}} \bar{V}_{s}(e) \tilde{\mu}(d s, d e)-\int_{t}^{T} d \bar{M}_{s}
\end{aligned}
$$

where

$$
\begin{aligned}
& \text { - } \varphi_{t}=\left(\bar{Y}_{t}\right)^{-1} \mathbb{1}_{\left\{\bar{Y}_{t} \neq 0\right\}}\left(F\left(t, Y_{t}^{1}, Z_{t}^{1}\right)-F\left(t, Y_{t}^{2}, Z_{t}^{1}\right)\right) \text {; } \\
& \text { - } \psi_{t}=\left(\bar{Z}_{t}\right)^{-1} \mathbb{1}_{\left\{\bar{Z}_{t} \neq 0\right\}}\left(F\left(t, Y_{t}^{2}, Z_{t}^{1}\right)-F\left(t, Y_{t}^{2}, Z_{t}^{2}\right)\right) \text {; } \\
& \text { - } \phi_{t}=f^{1}\left(t, Y_{t}^{2}, Z_{t}^{2}, V_{t}^{2}\right)-f^{2}\left(t, Y_{t}^{2}, Z_{t}^{2}, V_{t}^{2}\right) .
\end{aligned}
$$

Set

$$
\begin{aligned}
d \tilde{\mathbb{P}}= & {\left[\exp \left\{\int_{0}^{T} \psi_{s} d B_{s}-\int_{0}^{T} \frac{1}{2}\left|\psi_{s}\right|^{2} d s\right\} \prod_{0<t \leq T}\left(1+\int_{\mathcal{U}} \pi_{t}(\omega, e) \mu(\{t\}, d e)\right) \times\right.} \\
& \left.\exp \left\{-\int_{0}^{T} \int_{\mathcal{U}} \pi_{t}(\omega, e) \lambda(d e) d t\right\}\right] d \mathbb{P} .
\end{aligned}
$$

Then by Girsanov transformation theorem, there exists a probability measure $\tilde{\mathbb{P}}$ defined on the standard measurable space $(\Omega, \mathcal{F})$ such that where $\bar{B}_{t}=B_{t}-\int_{0}^{t} q_{s} d s$ is a Brownian motion under probability measure $\tilde{\mathbb{P}}$ and $\bar{\mu}(d t, d e)=\tilde{\mu}(d t, d e)-$ $\pi_{t}(\omega, e) \lambda(d e) d t$ is a $\tilde{\mathbb{P}}$-martingale measure. Then the DRBSDE (4.1) can be rewritten as

$$
\begin{aligned}
\bar{Y}_{t}= & \bar{\xi}_{T}+\int_{t}^{T}\left(\varphi_{s} \bar{Y}_{s}+\phi_{s}\right) d s+\left(\bar{K}_{T}^{+}-\bar{K}_{t}^{+}\right)-\left(\bar{K}_{T}^{-}-\bar{K}_{t}^{-}\right)-\int_{t}^{T} \bar{Z}_{s} d \bar{B}_{s} \\
& -\int_{t}^{T} \int_{\mathcal{U}} \bar{V}_{s}(e) \bar{\mu}(d s, d e)-\int_{t}^{T} d \bar{M}_{s} .
\end{aligned}
$$

From Proposition A.7, there exists a nondecreasing process $\left(\overline{\mathcal{A}}_{t}\right)_{t \leq T}$ with regulated trajectories such that

$$
\begin{aligned}
\left|\bar{Y}_{t}^{+}\right|^{2}= & \left|\bar{Y}_{0}^{+}\right|^{2}-2 \int_{0}^{t} \bar{Y}_{s}^{+}\left(\varphi_{s} \bar{Y}_{s}+\phi_{s}\right) d s-2 \int_{0}^{t} \bar{Y}_{s-}^{+} d \bar{K}_{s}^{+}+2 \int_{0}^{t} \bar{Y}_{s-}^{+} d \bar{K}_{s}^{-} \\
& +2 \int_{0}^{t} \bar{Y}_{s}^{+} \bar{Z}_{s} d \bar{B}_{s}+2 \int_{0}^{t} \int_{\mathcal{U}} \bar{Y}_{s-}^{+} \bar{V}_{s}(e) \bar{\mu}(d s, d e)+2 \int_{0}^{t} \bar{Y}_{s-}^{+} d \bar{M}_{s}+\overline{\mathcal{A}}_{t} .
\end{aligned}
$$


By applying the Corollary A.6 (see Appendix), we get

$$
\begin{aligned}
e^{\beta A_{t}}\left|\bar{Y}_{t}^{+}\right|^{2}+\beta \int_{t}^{T} e^{\beta A_{s}}\left|\bar{Y}_{s}^{+}\right|^{2} d A_{s}+\int_{t}^{T} e^{\beta A_{s}}\left|\bar{Z}_{s}\right|^{2} d s+\int_{t}^{T} e^{\beta A_{s}} d\langle\bar{M}\rangle_{s}^{c} \\
=e^{\beta A_{T}}\left|\bar{\xi}_{T}^{+}\right|^{2}+2 \int_{t}^{T} e^{\beta A_{s}} \bar{Y}_{s}^{+}\left(\varphi_{s} \bar{Y}_{s}+\phi_{s}\right) d s+2 \int_{t}^{T} e^{\beta A_{s}} \bar{Y}_{s-}^{+}\left(d \bar{K}_{s}^{+, *}-d \bar{K}_{s}^{-, *}\right) \\
\quad-2 \int_{t}^{T} e^{\beta A_{s}} \bar{Y}_{s}^{+} \bar{Z}_{s} d \bar{B}_{s}-2 \int_{t}^{T} \int_{\mathcal{U}} e^{\beta A_{s}} \bar{Y}_{s-}^{+} \bar{V}_{s}(e) \bar{\mu}(d s, d e)-2 \int_{t}^{T} e^{\beta A_{s}} \bar{Y}_{s}^{+} d \bar{M}_{s} \\
\quad-\sum_{t<s \leq T} e^{\beta A_{s}}\left(\bar{Y}_{s}^{+}-\bar{Y}_{s-}^{+}\right)^{2}-\sum_{t \leq s<T} e^{\beta A_{s}}\left(\left|\bar{Y}_{s+}^{+}\right|^{2}-\left|\bar{Y}_{s}^{+}\right|^{2}\right)-\int_{t}^{T} e^{\beta A_{s}} d \overline{\mathcal{A}}_{s} .
\end{aligned}
$$

Taking the $\tilde{\mathbb{P}}$-expectation on the both sides, taking into consideration the assumptions of theorem and using the facts that $\varphi_{s} \leq a_{s}^{2}$ and $\bar{Y}_{s-}^{+}\left(d \bar{K}_{s}^{+}-d \bar{K}_{s}^{-}\right) \leq 0$, we deduce for all $\beta>2$

$$
\mathbb{E}\left[e^{\beta A_{t}}\left|\bar{Y}_{t}^{+}\right|^{2}\right] \leq 0
$$

It follows that $\bar{Y}_{t}^{+}=0$, i.e. $Y_{t}^{1} \leq Y_{t}^{2}$ for all $t \leq T \tilde{\mathbb{P}}$-a.s. and so $\mathbb{P}$-a.s.

Remark 4.2. - If $\xi=-\infty$ then $d K^{+, j}=0$ for $j=1,2$ and the comparison theorem holds also for the upper-barrier reflected BSDEs.

- If $\zeta=+\infty$ then $d K^{-, j}=0$ for $j=1,2$ and the comparison theorem holds also for the lower-barrier reflected BSDEs.

- If $\xi=-\infty$ and $\zeta=+\infty$ then the comparison theorem holds also for the standard BSDEs.

Remark 4.3. If we consider the penalized equations relative to the RBSDE with data $\left(g^{j}, \xi^{j}\right)$ for $j=1,2$ and $n \in \mathbb{N}$, as follows

$$
\left\{\begin{array}{l}
Y_{t}^{n, j}=\xi_{T}^{j}+\int_{t}^{T} g^{j}(s) d s-n \int_{t}^{T}\left(Y_{s}^{n, j}-\zeta_{s}\right)^{+} d s-\sum_{t \leq \rho_{n, i}<T}\left(Y_{\rho_{n, i}+}^{n, j}-\zeta_{\rho_{n, i}}\right)^{+} \\
\quad+K_{T}^{+, n, j}-K_{t}^{+, n, j}-\int_{t}^{T} Z_{s}^{n, j} d B_{s}-\int_{t}^{T} \int_{\mathcal{U}} V_{s}^{n, j}(e) \tilde{\mu}(d s, d e)-\int_{t}^{T} d M_{s}^{n, j}, \\
Y_{t}^{n, j} \geq \xi_{t}^{j} \quad \forall t \leq T, \\
\int_{0}^{T}\left(Y_{t-}^{n, j}-\xi_{t-}^{j}\right) d K_{t}^{+, n, *, j}+\sum_{t<T}\left(Y_{t}^{n, j}-\xi_{t}^{j}\right) \Delta_{+} K_{t}^{+, n, j}=0 \text { a.s. }
\end{array}\right.
$$

Then, if $\xi^{1} \leq \xi^{2}$ and $g^{1} \leq g^{2}$, we have $K_{t}^{+, n, 1} \geq K_{t}^{+, n, 2}$. Actually, From Remark 4.2, we have $Y_{t}^{n, 1} \leq Y_{t}^{n, 2}$ for $t \leq T$. Since $K_{t}^{+, n, *, j}=n \int_{0}^{t}\left(Y_{s}^{n, j}-\xi_{s}^{j}\right)^{-} d s$ for $j=1$, we we deduce that $K_{t}^{+, n, *, 1} \geq K_{t}^{+, n, *, 2}$ for $t \leq T$. Similarly, we have $\sum_{s<t} \Delta_{+} K_{s}^{+, n, 1} \geq$ $\sum_{s<t} \Delta_{+} K_{s}^{+, n, 2}$ a.s.

\section{Appendix A.}

A.1. Special BSDEs in a general filtration. In this section we give a special case of existence and uniqueness result of BSDEs with jumps in a general filtration when the coefficient depends only on $y$. Consider the following BSDE

$$
Y_{t}=\xi+\int_{t}^{T} h\left(s, Y_{s}\right) d s-\int_{t}^{T} Z_{s} d B_{s}-\int_{t}^{T} \int_{\mathcal{U}} V_{s}(e) \tilde{\mu}(d s, d e)-\int_{t}^{T} d M_{s}
$$


where $\mathbb{E}\left[e^{\beta A_{T}}|\xi|^{2}\right]<+\infty, \frac{h(., 0)}{a} \in \mathcal{H}_{\beta}^{2}$ and $h$ is Lipschitz i.e. there exists a positive constant $\kappa$ such that for all $\left(t, y, y^{\prime}\right) \in[0, T] \times \mathbb{R}^{2},\left|h(t, y)-h\left(t, y^{\prime}\right)\right| \leq \kappa\left|y-y^{\prime}\right|$.

Theorem A.1. The BSDE (A.1) admits a unique solution $(Y, Z, V, M) \in \mathfrak{B}_{\beta}^{2} \times$ $\mathcal{H}_{\beta}^{2} \times \mathcal{L}_{\beta}^{2} \times \mathcal{M}_{\beta}^{2}$.

Proof: Remark that the condition $\mathbb{E} \int_{0}^{T}|h(t, 0)|^{2} d t<+\infty$ is not satisfied in our framework, then we can not apply the existence result of Kruse and Popier (2016). So, since

$$
\mathbb{E}\left|\xi+\int_{0}^{T} h(t, 0) d t\right|^{2} \leq 2 \mathbb{E} e^{\beta A_{T}}|\xi|^{2}+\frac{2}{\beta} \mathbb{E} \int_{0}^{T} e^{\beta A_{t}}\left|\frac{h(t, 0)}{a_{t}}\right|^{2} d t,
$$

then from the martingale representation Theorem (see Lemma 4.24, Chapter III in Jacod and Shiryaev, 2003), there exists a triplet of processes $(Z, V, M) \in \mathcal{H}^{2} \times \mathcal{L}^{2} \times$ $\mathcal{M}^{2}$ such that

$$
Y_{t}=\mathbb{E}\left[\xi+\int_{t}^{T} h(s, 0) d s\right]+\int_{0}^{t} Z_{s} d B_{s}+\int_{0}^{t} \int_{\mathcal{U}} V_{s}(e) \tilde{\mu}(d s, d e)+\int_{0}^{t} d M_{s}
$$

where $Y_{t}=\mathbb{E}\left[\xi+\int_{t}^{T} h(s, 0) d s \mid \mathcal{F}_{t}\right]$. Moreover, we have

$$
e^{\beta A_{t}}\left|Y_{t}\right|^{2} \leq 2 \mathbb{E}\left[e^{\beta A_{T}}|\xi|^{2}+\frac{1}{\beta} \int_{0}^{T} e^{\beta A_{s}}\left|\frac{h(s, 0)}{a_{s}}\right|^{2} d s \mid \mathcal{F}_{t}\right] .
$$

By Doob's maximal quadratic inequality, we deduce that

$$
\begin{aligned}
\mathbb{E}\left[\operatorname{lifs}_{\tau \in \mathcal{T}_{[0, T]}}^{\operatorname{essup}} e^{\beta A_{\tau}}\left|Y_{\tau}\right|^{2}\right] & =\mathbb{E}\left[\sup _{0 \leq t \leq T} e^{\beta A_{t}}\left|Y_{t}\right|^{2}\right] \\
& \leq 2 \mathbb{E}\left[e^{\beta A_{T}}|\xi|^{2}+\frac{1}{\beta} \int_{0}^{T} e^{\beta A_{s}}\left|\frac{h(s, 0)}{a_{s}}\right|^{2} d s\right] .
\end{aligned}
$$

On the other hand, by applying Itô's formula, we can find a positive constant $C$ such that

$$
\begin{aligned}
& \mathbb{E} \int_{t}^{T} e^{\beta A_{s}}\left|Y_{s}\right|^{2} d A_{s}+\mathbb{E} \int_{t}^{T} e^{\beta A_{s}}\left(\left|Z_{s}\right|^{2}+\left\|V_{s}\right\|_{\lambda}^{2}\right) d s+\mathbb{E} \int_{t}^{T} e^{\beta A_{s}} d[M]_{s} \\
\leq & C\left(\mathbb{E} e^{\beta A_{T}}|\xi|^{2}+\mathbb{E} \int_{0}^{T} e^{\beta A_{s}}\left|\frac{h(s, 0)}{a_{s}}\right|^{2} d s\right) .
\end{aligned}
$$

Next, define the sequence $\left(Y^{n}, Z^{n}, V^{n}\right)$ as follows: $\left(Y^{0}, Z^{0}, V^{0}\right)=(0,0,0)$ and $\left(Y^{n+1}, Z^{n+1}, V^{n+1}\right)$ is solution of the BSDE

$Y_{t}^{n+1}=\xi+\int_{t}^{T} h\left(s, Y_{s}^{n}\right) d s-\int_{t}^{T} Z_{s}^{n+1} d B_{s}-\int_{t}^{T} \int_{\mathcal{U}} V_{s}^{n+1}(e) \tilde{\mu}(d s, d e)-\int_{t}^{T} d M_{s}^{n+1}$.

For $n \geq p \geq 0$, let us put $\Re^{n, p}=\Re^{n}-\Re^{p}$ for $\Re \in\{Y, Z, V\}$. By using Itô's formula, we obtain

$$
\begin{aligned}
& \left\|Y^{n+1, p+1}\right\|_{\mathcal{S}_{\beta}^{2, A}}^{2}+\left\|Z^{n+1, p+1}\right\|_{\mathcal{H}_{\beta}^{2}}^{2}+\left\|V^{n+1, p+1}\right\|_{\mathcal{L}_{\beta}^{2}}^{2}+\left\|M^{n+1, p+1}\right\|_{\mathcal{M}_{\beta}^{2}}^{2} \\
\leq & \frac{\kappa^{2}}{\epsilon(\beta-1)}\left(\left\|Y^{n, p}\right\|_{\mathcal{S}_{\beta}^{2, A}}^{2}+\left\|Z^{n, p}\right\|_{\mathcal{H}_{\beta}^{2}}^{2}+\left\|V^{n, p}\right\|_{\mathcal{L}_{\beta}^{2}}^{2}+\left\|M^{n, p}\right\|_{\mathcal{M}_{\beta}^{2}}^{2}\right) .
\end{aligned}
$$


Choosing $\beta>0$ such that $\beta>\frac{\kappa^{2}}{\epsilon}+1$, then $\left(Y^{n}, Z^{n}, V^{n}, M^{n}\right)$ is a Cauchy sequence for the Banach space $\mathcal{S}_{\beta}^{2, A} \times \mathcal{H}_{\beta}^{2} \times \mathcal{L}_{\beta}^{2} \times \mathcal{M}_{\beta}^{2}$. Then, there exists a unique $(Y, Z, V, M) \in \mathcal{S}_{\beta}^{2, A} \times \mathcal{H}_{\beta}^{2} \times \mathcal{L}_{\beta}^{2} \times \mathcal{M}_{\beta}^{2}$ solution of BSDE (A.1).

In the following, a special comparison theorem for BSDE (A.1) that is a particular case of the Proposition 4 in Kruse and Popier (2016):

Theorem A.2. Let $\left(Y^{i}, Z^{i}, V^{i}, M^{i}\right)$ be a solution to BSDE (A.1) associated with parameters $\left(\xi^{i}, h^{i}\right)$ for $i=1,2$. If $\xi^{1} \leq \xi^{2}$ and $h^{1} \leq h^{2}$ then $Y^{1} \leq Y^{2}$ a.s.

A.2. Special RBSDEs in a general filtration. In the following, we prove the existence and uniqueness of solution to one lower barrier reflected BSDE with jumps and regulated trajectories which take the form:

$$
\left\{\begin{aligned}
Y_{t}= & \xi_{T}+\int_{t}^{T} h(s) d s+K_{T}-K_{t}-\int_{t}^{T} Z_{s} d B_{s}-\int_{t}^{T} \int_{\mathcal{U}} V_{s}(e) \tilde{\mu}(d s, d e) \\
& -\int_{t}^{T} d M_{s}, \\
Y_{t} \geq & \xi_{t} \quad \forall t \leq T \text { and } \int_{0}^{T}\left(Y_{t-}-\xi_{t-}\right) d K_{t}^{*}+\sum_{t<T}\left(Y_{t}-\xi_{t}\right) \Delta_{+} K_{t}=0 \text { a.s. }
\end{aligned}\right.
$$

where $\xi^{+} \in \mathcal{S}_{2 \beta}^{2}$ and $\frac{h}{a} \in \mathcal{H}_{\beta}^{2}$.

Theorem A.3. The RBSDE (A.Q) admits a unique solution $(Y, Z, V, M, K) \in$ $\mathfrak{B}_{\beta}^{2} \times \mathcal{H}_{\beta}^{2} \times \mathcal{L}_{\beta}^{2} \times \mathcal{M}_{\beta}^{2} \times \mathcal{S}^{2}$ and there exists a positive constant $C$ such that

$$
\begin{aligned}
& \mathbb{E} \operatorname{ess~sup}_{\tau \in \mathcal{T}_{[0, T]}} e^{\beta A_{\tau}}\left|Y_{\tau}\right|^{2}+\mathbb{E} \int_{t}^{T} e^{\beta A_{s}}\left|Y_{s}\right|^{2} d A_{s}+\mathbb{E} \int_{t}^{T} e^{\beta A_{s}}\left(\left|Z_{s}\right|^{2}+\left\|V_{s}\right\|_{\lambda}^{2}\right) d s \\
& +\mathbb{E} \int_{t}^{T} e^{\beta A_{s}} d[M]_{s}+\mathbb{E}\left|K_{T}\right|^{2} \\
\leq & C\left(\mathbb{E}\left[\operatorname{l}_{\tau \in \mathcal{T}_{[0, T]}}^{\operatorname{essup}} e^{2 \beta A_{\tau}}\left|\xi_{\tau}^{+}\right|^{2}\right]+\mathbb{E} \int_{0}^{T} e^{\beta A_{s}}\left|\frac{h(s)}{a_{s}}\right|^{2} d s\right)
\end{aligned}
$$

Proof: Here, we can not apply the existence result of Baadi and Ouknine (2017) since $\mathbb{E} \int_{0}^{T}|h(t)|^{2} d t<+\infty$ is not satisfy in general. So, for each $n \in \mathbb{N}$, we consider the following penalized version of BSDE

$$
\begin{aligned}
Y_{t}^{n}= & \xi_{T}+\int_{t}^{T} h(s) d s+n \int_{t}^{T}\left(Y_{s}^{n}-\xi_{s}\right)^{-} d s+\sum_{t \leq \sigma_{n, i}<T}\left(Y_{\sigma_{n, i}+}^{n}-\xi_{\sigma_{n, i}}\right)^{-} \\
& -\int_{t}^{T} Z_{s}^{n} d B_{s}-\int_{t}^{T} \int_{\mathcal{U}} V_{s}^{n}(e) \tilde{\mu}(d s, d e)-\int_{t}^{T} d M_{s}^{n}
\end{aligned}
$$

where $\left\{\sigma_{n, i}\right\}$ is arrays of stopping times exhausting right-jumps of $\xi$ defined, for all $n \in \mathbb{N}$, inductively by:

$$
\begin{cases}\sigma_{n, 0}=0, & i=1, \ldots, k_{1} \\ \sigma_{1, i}=\inf \left\{t>\sigma_{1, i-1} \mid \Delta_{+} \xi_{t}<-1\right\} \wedge T, & i=1, \ldots, j_{n+1}\end{cases}
$$


with $k_{1} \in \mathbb{N}$ and $j_{n+1}$ is chosen so that $\mathbb{P}\left(\sigma_{n+1, j_{n+1}}<T\right) \rightarrow 0$ as $n \rightarrow+\infty$ and

$$
\sigma_{n+1, i}=\sigma_{n+1, j_{n+1}} \vee \sigma_{n, i-j_{n+1}}, \quad i=j_{n+1}+1, \ldots, k_{n+1}, \quad k_{n+1}=j_{n+1}+k_{n} .
$$

Let $h^{n}(t, y)=h(t)+n\left(y-\xi_{t}\right)^{-}$. Remark that $h^{n}$ is $n$-Lipschitz and

$$
\mathbb{E} \int_{0}^{T} e^{\beta A_{t}}\left|\frac{h^{n}(t, 0)}{a_{t}}\right|^{2} d t \leq 2 \mathbb{E} \int_{0}^{T} e^{\beta A_{t}}\left|\frac{h(t)}{a_{t}}\right|^{2} d t+\frac{2 n^{2} T}{\epsilon} \underset{\tau \in \mathcal{T}_{[0, T]}}{\mathbb{E}} \operatorname{essup} e^{2 \beta A_{\tau}}\left|\xi_{\tau}^{+}\right|^{2} .
$$

Then, from Theorem A.1, on each interval $\left.] \sigma_{n, i-1}, \sigma_{n, i}\right], i=1, \ldots, k_{n}+1$ with $\sigma_{n, k_{n}+1}=T$, there exists a unique process $\left(Y^{n}, Z^{n}, V^{n}, M^{n}\right) \in \mathfrak{B}_{\beta}^{2} \times \mathcal{H}_{\beta}^{2} \times \mathcal{L}_{\beta}^{2} \times \mathcal{M}_{\beta}^{2}$ solution of the BSDE which take the form

$$
\begin{aligned}
Y_{t}^{n}= & \xi_{\sigma_{n, i}} \vee Y_{\sigma_{n, i}+}^{n}+\int_{t}^{\sigma_{n, i}} h(s) d s+n \int_{t}^{\sigma_{n, i}}\left(Y_{s}^{n}-\xi_{s}\right)^{-} d s-\int_{t}^{\sigma_{n, i}} Z_{s}^{n} d B_{s} \\
& \left.\left.-\int_{t}^{\sigma_{n, i}} \int_{\mathcal{U}} V_{s}^{n}(e) \tilde{\mu}(d s, d e)-\int_{t}^{\sigma_{n, i}} d M_{s}^{n}, \quad t \in\right] \sigma_{n, i-1}, \sigma_{n, i}\right]
\end{aligned}
$$

with the convention $Y_{T}^{n}=\xi_{T}$ and $Y_{0}^{n}=\xi_{0} \vee Y_{0+}^{n}$. On the other hand, the BSDE (A.4) can be written as

$Y_{t}^{n}=\xi_{T}+\int_{t}^{T} h(s) d s+K_{T}^{n}-K_{t}^{n}-\int_{t}^{T} Z_{s}^{n} d B_{s}-\int_{t}^{T} \int_{\mathcal{U}} V_{s}^{n}(e) \tilde{\mu}(d s, d e)-\int_{t}^{T} d M_{s}^{n}$

where

$$
K_{t}^{n}:=K_{t}^{n, *}+\sum_{s<t} \Delta_{+} K_{s}^{n}=n \int_{0}^{t}\left(Y_{s}^{n}-\xi_{s}\right)^{-} d s+\sum_{0 \leq \sigma_{n, i}<t}\left(Y_{\sigma_{n, i}+}^{n}-\xi_{\sigma_{n, i}}\right)^{-} .
$$

By applying Corollary A.6 and Burkholder-Davis-Gundy's inequality, we find that the sequence of processes $\left(Y^{n}, Z^{n}, V^{n}, M^{n}, K^{n}\right)_{n \geq 0}$ satisfies the uniform estimate

$$
\begin{aligned}
& \underset{\tau \in \mathcal{T}_{[0, T]}}{\mathbb{E} \operatorname{essup}} e^{\beta A_{\tau}}\left|Y_{\tau}^{n}\right|^{2}+\mathbb{E} \int_{0}^{T} e^{\beta A_{s}}\left|Y_{s}^{n}\right|^{2} d A_{s}+\mathbb{E} \int_{0}^{T} e^{\beta A_{s}}\left(\left|Z_{s}^{n}\right|^{2}+\left\|V_{s}^{n}\right\|_{\lambda}^{2}\right) d s \\
& +\mathbb{E} \int_{0}^{T} e^{\beta A_{s}} d\left[M^{n}\right]_{s}^{c}+\mathbb{E}\left|K_{T}^{n}\right|^{2} \\
& \leq C\left(\mathbb{E}\left[\operatorname{ess~sup}_{\tau \in \mathcal{T}_{[0, T]}}^{\operatorname{es} A_{\tau}}\left|\xi_{\tau}^{+}\right|^{2}\right]+\mathbb{E} \int_{0}^{T} e^{\beta A_{s}}\left|\frac{h(s)}{a_{s}}\right|^{2} d s\right)
\end{aligned}
$$

where $C$ is a positive constant independent of $n$.

Now, we establish the convergence of sequence $\left(Y^{n}, Z^{n}, V^{n}, M^{n}, K^{n}\right)$. Obviously that $h^{n}(., y) \leq h^{n+1}(., y)$ for each $n \in \mathbb{N}$, it follows from the comparison theorem A.2 that $Y^{n} \leq Y^{n+1}$. Hence there exists a process $Y$ such that $Y_{t}^{n} \nearrow Y_{t} \forall t \leq T$ a.s. and thanks to the monotonic limit theorem for regulated processes (see for instance Theorem 2.10 in Klimsiak et al., 2019) the limit process $Y$ has regulated trajectories. Moreover, From the uniform estimate (A.6) and Fatou's lemma, we have

$$
\begin{aligned}
& \mathbb{E}\left[\operatorname{esssup}_{\tau \in \mathcal{T}_{[0, T]}} e^{\beta A_{\tau}}\left|Y_{\tau}\right|^{2}\right] \leq \liminf _{n \rightarrow+\infty} \mathbb{E}\left[\operatorname{\tau ifs}_{\tau \in 0, T]}^{\operatorname{essup}} e^{\beta A_{\tau}}\left|Y_{\tau}^{n}\right|^{2}\right] \\
& \leq C\left(\mathbb{E}\left[\operatorname{esssup}_{\tau \in \mathcal{T}_{[0, T]}} e^{2 \beta A_{\tau}}\left|\xi_{\tau}^{+}\right|^{2}\right]+\mathbb{E} \int_{0}^{T} e^{\beta A_{s}}\left|\frac{h(s)}{a_{s}}\right|^{2} d s\right) .
\end{aligned}
$$


On the other hand, for all $n \geq p \geq 0$, Corollary A.6 implies

$$
\begin{aligned}
& e^{\beta A_{t}}\left|Y_{t}^{n}-Y_{t}^{p}\right|^{2}+\beta \int_{t}^{T} e^{\beta A_{s}}\left|Y_{s}^{n}-Y_{s}^{p}\right|^{2} d A_{s}+\int_{t}^{T} e^{\beta A_{s}}\left|Z_{s}^{n}-Z_{s}^{p}\right|^{2} d s \\
& +\int_{t}^{T} e^{\beta A_{s}} d\left\langle M^{n}-M^{p}\right\rangle_{s}^{c} \\
= & -2 \int_{t}^{T} e^{\beta A_{s}}\left(Y_{s}^{n}-Y_{s}^{p}\right)\left(Z_{s}^{n}-Z_{s}^{p}\right) d B_{s}-2 \int_{t}^{T} e^{\beta A_{s}}\left(Y_{s-}^{n}-Y_{s-}^{p}\right) d\left(M_{s}^{n}-M_{s}^{p}\right) \\
& -2 \int_{t}^{T} \int_{\mathcal{U}} e^{\beta A_{s}}\left(Y_{s-}^{n}-Y_{s-}^{p}\right)\left(V_{s}^{n}(e)-V_{s}^{p}(e)\right) \tilde{\mu}(d s, d e) \\
& +2 \int_{t}^{T} e^{\beta A_{s}}\left(Y_{s-}^{n}-Y_{s-}^{p}\right) d\left(K_{s}^{n, *}-K_{s}^{p, *}\right) \\
& -\sum_{t<s \leq T} e^{\beta A_{s}}\left|\Delta\left(Y_{s}^{n}-Y_{s}^{p}\right)\right|^{2}-\sum_{t \leq s<T} e^{\beta A_{s}}\left(\left|Y_{s+}^{n}-Y_{s+}^{p}\right|^{2}-\left|Y_{s}^{n}-Y_{s}^{p}\right|^{2}\right) .
\end{aligned}
$$

By using the same computations as those used to get (3.9), and the fact that

$$
\begin{aligned}
\left|Y_{s+}^{n}-Y_{s+}^{p}\right|^{2}-\left|Y_{s}^{n}-Y_{s}^{p}\right|^{2} & =\left|\Delta_{+}\left(Y_{s}^{n}-Y_{s}^{p}\right)\right|^{2}+2\left(Y_{s}^{n}-Y_{s}^{p}\right) \Delta_{+}\left(Y_{s}^{n}-Y_{s}^{p}\right) \\
& =\left|\Delta_{+}\left(Y_{s}^{n}-Y_{s}^{p}\right)\right|^{2}-2\left(Y_{s}^{n}-Y_{s}^{p}\right) \Delta_{+}\left(K_{s}^{n}-K_{s}^{p}\right)
\end{aligned}
$$

we obtain

$$
\begin{aligned}
& \beta \mathbb{E} \int_{0}^{T} e^{\beta A_{s}}\left|Y_{s}^{n}-Y_{s}^{p}\right|^{2} d A_{s}+\mathbb{E} \int_{0}^{T} e^{\beta A_{s}}\left(\left|Z_{s}^{n}-Z_{s}^{p}\right|^{2}+\left\|V_{s}^{n}-V_{s}^{p}\right\|_{\lambda}^{2}\right) d s \\
& +\mathbb{E} \int_{0}^{T} e^{\beta A_{s}} d\left[M^{n}-M^{p}\right]_{s} \\
\leq & 2 \mathbb{E} \int_{0}^{T} e^{\beta A_{s}}\left(Y_{s-}^{n}-Y_{s-}^{p}\right) d\left(K_{s}^{n}-K_{s}^{p}\right)-\mathbb{E} \sum_{0 \leq s<T} e^{\beta A_{s}}\left|\Delta_{+}\left(Y_{s}^{n}-Y_{s}^{p}\right)\right|^{2} \\
\leq & 2 \mathbb{E} \sup _{0 \leq t \leq T} e^{\beta A_{t}}\left(Y_{t-}^{p}-\xi_{t-}\right)^{-} K_{T}^{n}+2 \mathbb{E} \sup _{0 \leq t \leq T} e^{\beta A_{t}}\left(Y_{t-}^{n}-\xi_{t-}\right)^{-} K_{T}^{p} .
\end{aligned}
$$

By using the fact that

$$
\mathbb{E} \sup _{0 \leq t \leq T} e^{2 \beta A_{t}}\left|\left(Y_{t}^{n}-\xi_{t}\right)^{-}\right|^{2} \underset{n \rightarrow+\infty}{\longrightarrow} 0
$$

we can conclude that

$$
\left\|Y^{n}-Y^{p}\right\|_{\mathcal{S}_{\beta}^{2, A}}^{2}+\left\|Z^{n}-Z^{p}\right\|_{\mathcal{H}_{\beta}^{2}}^{2}+\left\|V^{n}-V^{p}\right\|_{\mathcal{L}_{\beta}^{2}}^{2}+\left\|M^{n}-M^{p}\right\|_{\mathcal{M}_{\beta}^{2}}^{2} \underset{n, p \rightarrow+\infty}{\longrightarrow} 0 .
$$

Then $\left(Y^{n}, Z^{n}, V^{n}, M^{n}\right)_{n \geq 0}$ is a Cauchy sequence in $\mathcal{S}_{\beta}^{2, A} \times \mathcal{H}_{\beta}^{2} \times \mathcal{L}_{\beta}^{2} \times \mathcal{M}_{\beta}^{2}$. So, there exists a quadruple $(Y, Z, V, M) \in \mathcal{S}_{\beta}^{2, A} \times \mathcal{H}_{\beta}^{2} \times \mathcal{L}_{\beta}^{2} \times \mathcal{M}_{\beta}^{2}$ such that

$$
\left\|Y^{n}-Y\right\|_{\mathcal{S}_{\beta}^{2, A}}^{2}+\left\|Z^{n}-Z\right\|_{\mathcal{H}_{\beta}^{2}}^{2}+\left\|V^{n}-V\right\|_{\mathcal{L}_{\beta}^{2}}^{2}+\left\|M^{n}-M\right\|_{\mathcal{M}_{\beta}^{2}}^{2} \underset{n \rightarrow+\infty}{\longrightarrow} 0 .
$$

On the other hand, by Burkholder-Davis-Gundy's inequality, we have

$$
\mathbb{E}\left[\operatorname{ess}_{\tau \in \mathcal{T}_{[0, T]}} e^{\beta A_{\tau}}\left|Y_{\tau}^{n}-Y_{\tau}^{p}\right|^{2}\right] \underset{n, p \rightarrow+\infty}{\longrightarrow} 0 .
$$




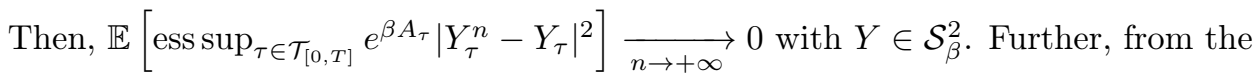
equation (A.5), we have also

$$
\mathbb{E}\left[\operatorname{ess~sup}_{\tau \in \mathcal{T}_{[0, T]}}^{\operatorname{en}}\left|K_{\tau}^{n}-K_{\tau}^{p}\right|^{2}\right] \underset{n, p \rightarrow+\infty}{\longrightarrow} 0 .
$$

Consequently, there exists a optional process $K$ limit of $K^{n}$ as $n \longrightarrow+\infty$. It remains to check the Skorokhod condition. We have just seen that the sequence $\left(Y^{n}, K^{n}\right)$ tends to $(Y, K)$ uniformly in $t$ in probability with $K=K^{*}+\Delta_{+} K$ with $\left.\Delta_{+} K_{t}=\left(Y_{t+}-\xi_{t}\right)^{-} \mathbb{1}_{\left\{Y_{t}=\xi_{t}\right\}}\right)$. Then the measure $d K^{n, *}$ tends to $d K^{*}$ weakly in probability, hence

$$
\int_{0}^{T}\left(Y_{t}^{n}-\xi_{t}\right) d K_{t}^{n, *} \underset{n \rightarrow+\infty}{\stackrel{\mathbb{P}}{\longrightarrow}} \int_{0}^{T}\left(Y_{t}-\xi_{t}\right) d K_{t}^{*}
$$

We deduce from the equation (A.7) that $\int_{0}^{T}\left(Y_{t}^{n}-\xi_{t}\right) d K_{t}^{n, *} \leq 0, n \in \mathbb{N}$, which implies that $\int_{0}^{T}\left(Y_{t}-\xi_{t}\right) d K_{t}^{*} \leq 0$. On the other hand, since $Y_{t} \geq \xi_{t}$, we have $\int_{0}^{T}\left(Y_{t}-\xi_{t}\right) d K_{t}^{*} \geq 0$. Hence $\int_{0}^{T}\left(Y_{t}-\xi_{t}\right) d K_{t}^{*}=0$. Finally, by applying Corollary A.6, one can derive that

$$
\begin{aligned}
& \mathbb{E} \int_{0}^{T} e^{\beta A_{s}}\left|Y_{s}\right|^{2} d A_{s}+\mathbb{E} \int_{0}^{T} e^{\beta A_{s}}\left(\left|Z_{s}\right|^{2}+\left\|V_{s}\right\|_{\lambda}^{2}\right) d s+\mathbb{E} \int_{0}^{T} e^{\beta A_{s}} d[M]_{s}+\mathbb{E}\left|K_{T}\right|^{2}
\end{aligned}
$$

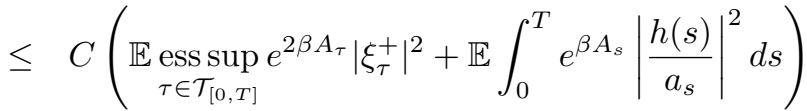

where $C$ is a positive constant. The proof is complete.

Corollary A.4. The RBSDE with one upper reflecting barrier $\zeta$, that is

$$
\left\{\begin{aligned}
& Y_{t}= \zeta_{T}+\int_{t}^{T} h(s) d s-K_{T}+K_{t}-\int_{t}^{T} Z_{s} d B_{s}-\int_{t}^{T} \int_{\mathcal{U}} V_{s}(e) \tilde{\mu}(d s, d e) \\
& \quad-\int_{t}^{T} d M_{s}, \\
& Y_{t} \leq \zeta_{t} \quad \forall t \leq T \text { and } \int_{0}^{T}\left(\zeta_{t-}-Y_{t-}\right) d K_{t}^{*}+\sum_{t<T}\left(\zeta_{t}-Y_{t}\right) \Delta_{+} K_{t}=0 \text { a.s. }
\end{aligned}\right.
$$

admits a unique solution $(Y, Z, V, M, K) \in \mathfrak{B}_{\beta}^{2} \times \mathcal{H}_{\beta}^{2} \times \mathcal{L}_{\beta}^{2} \times \mathcal{M}_{\beta}^{2} \times \mathcal{S}^{2}$ and there exists a positive constant $C$ such that

$$
\begin{aligned}
& \mathbb{E}\left[\operatorname{esssup}_{\tau \in \mathcal{T}_{[0, T]}} e^{\beta A_{\tau}}\left|Y_{\tau}\right|^{2}\right]+\mathbb{E} \int_{t}^{T} e^{\beta A_{s}}\left|Y_{s}\right|^{2} d A_{s}+\mathbb{E} \int_{t}^{T} e^{\beta A_{s}}\left(\left|Z_{s}\right|^{2}+\left\|V_{s}\right\|_{\lambda}^{2}\right) d s \\
& +\mathbb{E} \int_{t}^{T} e^{\beta A_{s}} d[M]_{s}+\mathbb{E}\left|K_{T}\right|^{2} \\
& \leq C\left(\mathbb{E}\left[\operatorname{esssup}_{\tau \in \mathcal{T}_{[0, T]}} e^{2 \beta A_{\tau}}\left|\zeta_{\tau}^{-}\right|^{2}\right]+\mathbb{E} \int_{0}^{T} e^{\beta A_{s}}\left|\frac{h(s)}{a_{s}}\right|^{2} d s\right) .
\end{aligned}
$$


A.3. Itô's formula for irregular processes.

Theorem A.5. Let $Y$ be an adapted process with regulated trajectories such that

$$
Y_{t}=Y_{t}^{*}+\sum_{s<t} \Delta_{+} Y_{s} \quad \forall t \leq T
$$

where $Y^{*}$ is the càdlàg part of the process $Y$ and $\sum_{s<t}\left|\Delta_{+} Y_{s}\right|<+\infty$ a.s. Let $F$ be a twice continuously differentiable function on $\mathbb{R}^{n}$. Then, almost surely, for each $n \in \mathbb{N}$ and all $t \geq 0$,

$$
\begin{aligned}
& F\left(Y_{t}\right) \\
= & F\left(Y_{0}\right)+\sum_{k=1}^{n} \int_{0}^{t} D^{k} F\left(Y_{s-}\right) d Y_{s}^{*, k}+\frac{1}{2} \sum_{k, l=1}^{n} \int_{0}^{t} D^{k} D^{l} F\left(Y_{s-}\right) d\left[Y^{*, k}, Y^{*, l}\right]_{s}^{c} \\
& +\sum_{0<s \leq t}\left[F\left(Y_{s}\right)-F\left(Y_{s-}\right)-\sum_{k=1}^{n} D^{k} F\left(Y_{s-}\right) \Delta Y_{s}^{k}\right]+\sum_{0 \leq s<t}\left[F\left(Y_{s+}\right)-F\left(Y_{s}\right)\right],
\end{aligned}
$$

where $D^{k}$ denotes the differentiation operator with respect to the $k$-th coordinate, and $[., .]^{c}$ denotes the continuous part of the quadratic variation of corresponding process.

Corollary A.6. Let $Y$ be an adapted process with regulated trajectories and $X$ be a continuous process of finite variation. Then, almost surely, for all $t \geq 0$,

$$
\begin{aligned}
& F\left(X_{t}, Y_{t}\right)=F\left(X_{0}, Y_{0}\right)+\int_{0}^{t} \partial_{X} F\left(X_{s}, Y_{s}\right) d s+\int_{0}^{t} \partial_{Y} F\left(X_{s}, Y_{s-}\right) d Y_{s}^{*} \\
& +\frac{1}{2} \int_{0}^{t} \partial_{Y}^{2} F\left(X_{s}, Y_{s-}\right) d\left\langle Y^{*}\right\rangle_{s}^{c}+\sum_{0<s \leq t}\left[F\left(X_{s}, Y_{s}\right)-F\left(X_{s}, Y_{s-}\right)-\partial_{Y} F\left(X_{s}, Y_{s-}\right) \Delta Y_{s}\right] \\
& +\sum_{0 \leq s<t}\left[F\left(X_{s}, Y_{s+}\right)-F\left(X_{s}, Y_{s}\right)\right]
\end{aligned}
$$

where $\partial_{Y}$ is the partial derivative operator with respect to $Y$.

In what follows, we give a version of Tanaka's formula of a strong optional semimartingales which can be seen as an extension of theorem 66 page 210 in Protter (2005)

Proposition A.7 (Tanaka's formula). Let $Y$ be an adapted process with regulated trajectories and $F: \mathbb{R} \rightarrow \mathbb{R}$ be a convex function. Then, $f(Y)$ is a strong optional semimartingale. Moreover, denoting by $F^{\prime}$ the left-hand derivative of the convex function $F$. Then, almost surely, for each $n \in \mathbb{N}$ and all $t \geq 0$,

$$
F\left(Y_{t}\right)=F\left(Y_{0}\right)+\int_{0}^{t} F^{\prime}\left(Y_{s-}\right) d Y_{s}^{*}+\mathcal{A}_{t}
$$

where $\mathcal{A}$ is a nondecreasing adapted process with regulated trajectories such that

$$
\Delta \mathcal{A}_{t}=F\left(Y_{t}\right)-F\left(Y_{t-}\right)-F^{\prime}\left(Y_{t-}\right) \Delta Y_{t} \quad \text { and } \quad \Delta_{+} \mathcal{A}_{t}=F\left(Y_{t+}\right)-F\left(Y_{t}\right) .
$$

Proof: See the proof of Lemma 9.1 in Grigorova et al. (2020). 


\section{Acknowledgements}

The authors would like to thank the referee for the careful reading of the paper and highly appreciate the comments and suggestions, which significantly contributed to improving the quality of the paper.

\section{References}

Akdim, K., Haddadi, M., and Ouknine, Y. Strong snell envelopes and RBSDEs with regulated trajectories when the barrier is a semimartingale. Stochastics, 92 (3), 335-355 (2020). MR4085755.

Arharas, I., Bouhadou, S., and Ouknine, Y. Doubly Reflected Backward Stochastic Differential Equations in the Predictable Setting. Journal of Theoretical Probability, pp. 1-27 (2021). DOI: 10.1007/s10959-020-01070-5.

Baadi, B. and Ouknine, Y. Reflected BSDEs when the obstacle is not rightcontinuous in a general filtration. ALEA Lat. Am. J. Probab. Math. Stat., 14 (1), 201-218 (2017). MR3655127.

Baadi, B. and Ouknine, Y. Reflected BSDEs with optional barrier in a general filtration. Afr. Mat., 29 (7-8), 1049-1064 (2018). MR3869547.

Bahlali, K., Hamadène, S., and Mezerdi, B. Backward stochastic differential equations with two reflecting barriers and continuous with quadratic growth coefficient. Stochastic Process. Appl., 115 (7), 1107-1129 (2005). MR2147243.

Barles, G., Buckdahn, R., and Pardoux, E. Backward stochastic differential equations and integral-partial differential equations. Stochastics Stochastics Rep., 60 (1-2), 57-83 (1997). MR1436432.

Bismut, J.-M. Conjugate convex functions in optimal stochastic control. J. Math. Anal. Appl., 44, 384-404 (1973). MR329726.

Cvitanić, J. and Karatzas, I. Backward stochastic differential equations with reflection and Dynkin games. Ann. Probab., 24 (4), 2024-2056 (1996). MR1415239.

El Karoui, N. Les aspects probabilistes du contrôle stochastique. In Ninth Saint Flour Probability Summer School-1979 (Saint Flour, 1979), volume 876 of Lecture Notes in Math., pp. 73-238. Springer, Berlin-New York (1981). MR637471.

El Karoui, N. and Huang, S.-J. A general result of existence and uniqueness of backward stochastic differential equations. In Backward stochastic differential equations (Paris, 1995-1996), volume 364 of Pitman Res. Notes Math. Ser., pp. 27-36. Longman, Harlow (1997). MR1752673.

El Karoui, N., Kapoudjian, C., Pardoux, E., Peng, S., and Quenez, M. C. Reflected solutions of backward SDE's, and related obstacle problems for PDE's. Ann. Probab., 25 (2), 702-737 (1997a). MR1434123.

El Karoui, N., Pardoux, E., and Quenez, M. C. Reflected backward SDEs and American options. In Numerical methods in finance, volume 13 of Publ. Newton Inst., pp. 215-231. Cambridge Univ. Press, Cambridge (1997b). MR1470516.

El Karoui, N., Peng, S., and Quenez, M. C. Backward stochastic differential equations in finance. Math. Finance, 7 (1), 1-71 (1997c). MR1434407.

El Karoui, N. and Quenez, M. C. Non-linear pricing theory and backward stochastic differential equations. In Financial mathematics (Bressanone, 1996), volume 1656 of Lecture Notes in Math., pp. 191-246. Springer, Berlin (1997). MR1478202.

Essaky, E. H. Reflected backward stochastic differential equation with jumps and RCLL obstacle. Bull. Sci. Math., 132 (8), 690-710 (2008). MR2474488. 
Essaky, E. H., Ouknine, Y., and Harraj, N. Backward stochastic differential equations with two reflecting barriers and jumps. Stoch. Anal. Appl., 23 (5), 921-938 (2005). MR2158885.

Grigorova, M., Imkeller, P., Offen, E., Ouknine, Y., and Quenez, M.-C. Reflected BSDEs when the obstacle is not right-continuous and optimal stopping. Ann. Appl. Probab., 27 (5), 3153-3188 (2017). MR3719955.

Grigorova, M., Imkeller, P., Ouknine, Y., and Quenez, M.-C. Doubly reflected BSDEs and $\mathcal{E}^{f}$-Dynkin games: beyond the right-continuous case. Electron. J. Probab., 23, Paper No. 122, 38 (2018). MR3896859.

Grigorova, M., Imkeller, P., Ouknine, Y., and Quenez, M.-C. Optimal stopping with f-expectations: the irregular case. Stochastic Process. Appl., 130 (3), 1258-1288 (2020). MR4058273.

Hamadène, S. Reflected BSDE's with discontinuous barrier and application. Stoch. Stoch. Rep., 74 (3-4), 571-596 (2002). MR1943580.

Hamadène, S. and Hassani, M. BSDEs with two reflecting barriers: the general result. Probab. Theory Related Fields, 132 (2), 237-264 (2005). MR2199292.

Hamadène, S. and Hassani, M. BSDEs with two reflecting barriers driven by a Brownian and a Poisson noise and related Dynkin game. Electron. J. Probab., 11, no. 5, 121-145 (2006). MR2217812.

Hamadène, S. and Lepeltier, J.-P. Reflected BSDEs and mixed game problem. Stochastic Process. Appl., 85 (2), 177-188 (2000). MR1731020.

Hamadène, S. and Ouknine, Y. Reflected backward stochastic differential equation with jumps and random obstacle. Electron. J. Probab., 8, no. 2, 20 (2003). MR1961164.

Hamadène, S. and Ouknine, Y. Reflected backward SDEs with general jumps. Theory Probab. Appl., 60 (2), 263-280 (2016). MR3568776.

Hamadène, S. and Wang, H. BSDEs with two RCLL reflecting obstacles driven by Brownian motion and Poisson measure and a related mixed zero-sum game. Stochastic Process. Appl., 119 (9), 2881-2912 (2009). MR2554032.

Jacod, J. Calcul stochastique et problèmes de martingales, volume 714 of Lecture Notes in Mathematics. Springer, Berlin (1979). ISBN 3-540-09253-6. MR542115.

Jacod, J. and Shiryaev, A. N. Limit theorems for stochastic processes, volume 288 of Grundlehren der Mathematischen Wissenschaften /Fundamental Principles of Mathematical Sciences/. Springer-Verlag, Berlin, second edition (2003). ISBN 3-540-43932-3. MR1943877.

Klimsiak, T., Rzymowski, M., and Słomiński, L. Reflected BSDEs with regulated trajectories. Stochastic Process. Appl., 129 (4), 1153-1184 (2019). MR3926552.

Klimsiak, T., Rzymowski, M., and Słomiński, L. Reflected backward stochastic differential equations with two optional barriers. Bull. Sci. Math., 158, 102820, 49 (2020). MR4030244.

Kruse, T. and Popier, A. BSDEs with monotone generator driven by Brownian and Poisson noises in a general filtration. Stochastics, 88 (4), 491-539 (2016). MR3473849.

Li, M. and Shi, Y. Solving the double barrier reflected BSDEs via penalization method. Statist. Probab. Lett., 110, 74-83 (2016). MR3474740.

Marzougue, M. A note on optional Snell envelopes and reflected backward SDEs. Statist. Probab. Lett., 165, 108833,7 (2020). MR4113844. 
Marzougue, M. and El Otmani, M. Double barrier reflected BSDEs with stochastic Lipschitz coefficient. Mod. Stoch. Theory Appl., 4 (4), 353-379 (2017). MR3739014.

Marzougue, M. and El Otmani, M. Non-continuous double barrier reflected BSDEs with jumps under a stochastic Lipschitz coefficient. Commun. Stoch. Anal., 12 (4), Art. 1, 359-381 (2018). MR3957705.

Marzougue, M. and El Otmani, M. BSDEs with right upper-semicontinuous reflecting obstacle and stochastic Lipschitz coefficient. Random Oper. Stoch. Equ., 27 (1), 27-41 (2019). MR3916509.

Marzougue, M. and El Otmani, M. Predictable solution for reflected BSDEs when the obstacle is not right-continuous. Random Oper. Stoch. Equ., 28 (4), 269-279 (2020a). MR4173846.

Marzougue, M. and El Otmani, M. Reflected BSDEs with jumps and two rcll barriers under stochastic Lipschitz coefficient. Communications in Statistics Theory and Methods (2020b). DOI: 10.1080/03610926.2020.1738491.

Pardoux, E. and Peng, S. Backward stochastic differential equations and quasilinear parabolic partial differential equations. In Stochastic partial differential equations and their applications (Charlotte, NC, 1991), volume 176 of Lect. Notes Control Inf. Sci., pp. 200-217. Springer, Berlin (1992). MR1176785.

Pardoux, E. and Peng, S. G. Adapted solution of a backward stochastic differential equation. Systems Control Lett., 14 (1), 55-61 (1990). MR1037747.

Protter, P. E. Stochastic integration and differential equations, volume 21 of Stochastic Modelling and Applied Probability. Springer-Verlag, Berlin (2005). ISBN 3-540-00313-4. MR2273672.

Quenez, M.-C. and Sulem, A. Reflected BSDEs and robust optimal stopping for dynamic risk measures with jumps. Stochastic Process. Appl., 124 (9), 3031-3054 (2014). MR3217432.

Royer, M. Backward stochastic differential equations with jumps and related non-linear expectations. Stochastic Process. Appl., 116 (10), 1358-1376 (2006). MR2260739.

Yin, J. and Mao, X. The adapted solution and comparison theorem for backward stochastic differential equations with Poisson jumps and applications. J. Math. Anal. Appl., 346 (2), 345-358 (2008). MR2431531. 